\title{
REPRESENTATIONS OF $p$-CONVOLUTION ALGEBRAS ON $L^{q}$-SPACES
}

\author{
EUSEBIO GARDELLA AND HANNES THIEL
}

\begin{abstract}
For a nontrivial locally compact group $G$, consider the Banach algebras of $p$-pseudofunctions, $p$-pseudomeasures, $p$-convolvers, and the full group $L^{p}$-operator algebra. We show that these Banach algebras are operator algebras if and only if $p=2$. More generally, we show that for $q \in[1, \infty)$, these Banach algebras can be represented on an $L^{q}$-space if and only if one of the following holds: (a) $p=2$ and $G$ is abelian; or (b) $\left|\frac{1}{p}-\frac{1}{2}\right|=\left|\frac{1}{q}-\frac{1}{2}\right|$. This result can be interpreted as follows: for $p, q \in[1, \infty)$, the $L^{p}$ - and $L^{q}$ representation theories of a group are incomparable, except in the trivial cases when they are equivalent.

As an application, we show that, for distinct $p, q \in[1, \infty)$, if the $L^{p}$ and $L^{q}$ crossed products of a topological dynamical system are isomorphic, then $\frac{1}{p}+\frac{1}{q}=1$. In order to prove this, we study the following relevant aspects of $L^{p}$-crossed products: existence of approximate identities, duality with respect to $p$, and existence of canonical isometric maps from group algebras into their multiplier algebras.
\end{abstract}

\section{Contents}

1. Introduction

2. Preliminaries

3. $\quad F^{p}(\mathbb{Z})$ is not isomorphic to $F^{q}(\mathbb{Z})$

4. Nonrepresentability on $L^{q}$-spaces

5. An application to crossed products

References

Date: 17 July 2018.

2010 Mathematics Subject Classification. Primary: 47L10, 43A15, Secondary: 43A65, 46E30.

Key words and phrases. Locally compact group, algebra of $p$-pseudofunctions, algebra of $p$ pseudomeasures, contractive approximate identity, multiplier algebra, group amenability, crossed product.

The first named author was partially supported by the D. K. Harrison Prize from the University of Oregon and by a Postdoctoral Research Fellowship from the Humboldt Foundation. The second named author was partially supported by the Deutsche Forschungsgemeinschaft (SFB 878). Part of this work was completed while the authors were taking part in the Research Program Classification of operator algebras, complexity, rigidity and dynamics, held at the Institut Mittag-Leffler, between January and April of 2016. We would like to thank the staff and organizers, and Søren Eilers in particular, for the hospitality and financial support. 


\section{INTRODUCTION}

We say that a Banach algebra is an operator algebra if it admits an isometric representation as bounded operators on a Hilbert space. Associated to any locally compact group $G$ there are three fundamentally important operator algebras: its reduced group $C^{*}$-algebra $C_{\lambda}^{*}(G)$, its full group $C^{*}$-algebra $C^{*}(G)$, and its group von Neumann algebra $L(G)$. These are, respectively, the Banach algebra generated by the left regular representation of $G$ on $L^{2}(G)$; the universal $C^{*}$-algebra with respect to unitary representations of $G$ on Hilbert spaces; and the weak* closure (also called ultraweak closure) of $C_{\lambda}^{*}(G)$ in $\mathcal{B}\left(L^{2}(G)\right.$ ). (We canonically identify $\mathcal{B}\left(L^{2}(G)\right.$ ) with the dual of the projective tensor product $L^{2}(G) \widehat{\otimes} L^{2}(G)$.) Equivalently, $L(G)$ is the double commutant of $C_{\lambda}^{*}(G)$ in $\mathcal{B}\left(L^{2}(G)\right)$.

These operator algebras admit generalizations to representations of $G$ on $L^{p}$ spaces, for $p \in[1, \infty)$. The analog of $C_{\lambda}^{*}(G)$ is the algebra $F_{\lambda}^{p}(G)$ of $p$-pseudofunctions on $G$, introduced by Herz in Her73 and originally denoted by $P F_{p}(G)$. The analog of $C^{*}(G)$ is the full group $L^{p}$-operator algebra $F^{p}(G)$, defined by Phillips in Phi13. Finally, the von Neumann algebra $L(G)$ has two analogs, at least for $p \neq 1$ : the algebra $P M_{p}(G)$ of $p$-pseudomeasures, which is the weak* closure of $F_{\lambda}^{p}(G)$ in $\mathcal{B}\left(L^{p}(G)\right)$ (where we canonically identify $\mathcal{B}\left(L^{p}(G)\right.$ ) with the dual of the projective tensor product $\left.L^{p}(G) \widehat{\otimes} L^{p}(G)^{*}\right)$; and the algebra $C V_{p}(G)$ of $p$-convolvers, which is the double commutant of $F_{\lambda}^{p}(G)$ in $\mathcal{B}\left(L^{p}(G)\right.$ ) (it is also the commutant of the right regular representation). Both $P M_{p}(G)$ and $C V_{p}(G)$ were introduced in Her73.

These objects, and related ones, have been studied by a number of authors in the last three decades. For instance, see Cow98, NR09, Run05, DS13, and the more recent papers [Phi13, [Phi14, GT15b], and [GT14. In GT16a], it is shown that there is a certain quotient of $F^{p}(\mathbb{Z})$ which cannot be represented on an $L^{p}$-space (in fact, on any $L^{q}$-space for $q \in[1, \infty)$ ), thus answering a 20 -year-old question of Le Merdy.

Despite the advances in the area, some basic questions remain open. One important open problem is whether $P M_{p}(G)=C V_{p}(G)$ for all $p \in(1, \infty)$ and for all locally compact groups $G$. This is known to be true when $p=2$ (both algebras agree with $L(G)$ ), essentially by the double commutant theorem. It is immediate that $P M_{p}(G) \subseteq C V_{p}(G)$ in general, while Herz showed in Her73 that equality holds for all $p$ if $G$ is amenable, a result that was later generalized by Cowling in Cow98 to groups with the approximation property.

A less studied problem is the following. By universality of $F^{p}(G)$, there is a canonical contractive homomorphism $\kappa_{p}: F^{p}(G) \rightarrow F_{\lambda}^{p}(G)$ with dense range. For $p=2$, this map is known to be a quotient map, and for $p=1$ it is an isomorphism regardless of $G$. On the other hand, we do not know if $\kappa_{p}$ is also a quotient map for all other values of $p$. In fact, we do not even know whether $\kappa_{p}$ is surjective. If this map is not necessarily surjective, can it be injective without the group being amenable? (By Theorem 3.7 in GT15b, $G$ is amenable if and only if $\kappa_{p}$ is bijective for some (equivalently, for all) $p \in(1, \infty)$. This result was independently obtained by Phillips in Phi13] and Phi14, using different methods.) In this case, it would be interesting to describe precisely for what groups (and Hölder exponents) the map $\kappa_{p}$ is injective but not surjective.

Questions of the nature described above would in principle be easier to tackle if the objects considered had a better understood structure, as is the case for operator algebras. Despite the fact that the Banach algebras $F^{p}(G), F_{\lambda}^{p}(G), P M_{p}(G)$ and 
$C V_{p}(G)$ have natural representations as operators on an $L^{p}$-space, this by itself does not rule out having isometric representations on Hilbert spaces as well; see, for example, BLM95. It is therefore not a priori clear whether the $L^{p}$-analogs of group operator algebras can be isometrically represented on Hilbert spaces.

In this paper, we settle this question negatively. Indeed, we show in Theorem 4.12 that for a nontrivial locally compact group $G$, and for $p \in[1, \infty) \backslash\{2\}$, none of the algebras $F^{p}(G), F_{\lambda}^{p}(G), P M_{p}(G)$, or $C V_{p}(G)$ can be isometrically represented on a Hilbert space. This result generalizes Theorem 2.2 in [NR09, where Neufang and Runde assume that $G$ is amenable and has a closed infinite abelian subgroup. More generally, for $p, q \in[1, \infty)$ we show that the algebras $F_{\lambda}^{p}(G), F^{p}(G), P M_{p}(G)$, or $C V_{p}(G)$ can be isometrically represented on an $L^{q}$-space if and only if one of the following holds:

(1) $p=2$ and $G$ is abelian; or

(2) $\left|\frac{1}{p}-\frac{1}{2}\right|=\left|\frac{1}{q}-\frac{1}{2}\right|$. (This is equivalent to either $p=q$ or $\frac{1}{p}+\frac{1}{q}=1$.)

This result can be interpreted as asserting that the $L^{p}$ - and $L^{q}$-representation theories of a nontrivial group are incomparable, whenever they are not "obviously" equivalent. As a consequence, it follows that if there is an isometric Banach algebra isomorphism $F_{\lambda}^{p}(G) \cong F_{\lambda}^{q}(G)$ (or between full group algebras, pseudomeasures or convolvers) for distinct $p, q \in[1, \infty)$, then $\frac{1}{p}+\frac{1}{q}=1$; see Corollary 4.13. The converse also holds; see Theorem 4.11.

As an application, we show that, for $p, q \in[1, \infty)$, if the $L^{p}$ - and $L^{q}$-crossed products of a topological dynamical system are isometrically isomorphic, then $\left|\frac{1}{p}-\frac{1}{2}\right|=\left|\frac{1}{q}-\frac{1}{2}\right|$; see Corollary 5.11. Since we do not know whether an isomorphism $F^{p}(G, X, \alpha) \rightarrow F^{q}(G, X, \alpha)$ (or $F_{\lambda}^{p}(G, X, \alpha) \rightarrow F_{\lambda}^{q}(G, X, \alpha)$ ) must necessarily respect the group action $\alpha$, Corollary 4.13 is not enough to obtain the conclusion. This means that even if we are only interested in isomorphisms of crossed products, we are forced to consider arbitrary representations of $p$-convolution algebras on $L^{q}$-spaces. In order to obtain these results, we need to develop the theory of $L^{p}$-crossed products further, and we do so by exploring the following fundamental aspects: existence of approximate identities, duality with respect to $p$, and existence of canonical isometric maps from group algebras into their multiplier algebras.

We give an outline of the proof of our main result (Theorem 4.12). Let $E$ be an $L^{q}$-space and let $\varphi: F_{\lambda}^{p}(G) \rightarrow \mathcal{B}(E)$ be an isometric representation. (Similar arguments apply for the algebras $F^{p}(G), P M_{p}(G)$, or $C V_{p}(G)$.)

Step 1 . The case $q=2$ is treated separately, since in this case one can show that $F_{\lambda}^{p}(G)$ is a $C^{*}$-algebra; see Theorem 4.6. So assume that $q \neq 2$.

Step 2. Using results in GT16c, we may assume that $\varphi$ is non-degenerate.

Step 3. Functoriality properties of $F_{\lambda}^{p}$ with respect to subgroups (GT14) allow us to further reduce the problem to the case where $G$ is a cyclic group (finite or infinite); see Lemma 4.10.

Step 4. For cyclic $G$, we need to know that $\varphi\left(F_{\lambda}^{p}(G)\right)$ is isometrically isomorphic to $F_{\lambda}^{q}(G)$. This requires non-trivial results from [GT15a] on spectral configurations. (For instance, we use the fact that $F_{\lambda}^{q}(\mathbb{Z})$ is the unique $L^{q}$ operator algebra generated by an invertible isometry whose Gelfand transform is not surjective.) We conclude that there is an isometric isomorphism $F_{\lambda}^{p}(G) \cong F_{\lambda}^{q}(G)$. 
Step 5. When $G=\mathbb{Z}$, we show that $\left|\frac{1}{p}-\frac{1}{2}\right|=\left|\frac{1}{q}-\frac{1}{2}\right|$ in Theorem 3.6 using elementary computations.

Step 6. When $G=\mathbb{Z}_{n}$, we use the existence, for $1 \leq p \leq q \leq 2$, of a canonical, contractive map $\gamma_{p, q}: F^{p}(G) \rightarrow F^{q}(G)$ with dense range (see Theorem 2.5), to show that the Gelfand transform of $F_{\lambda}^{p}(G)$ is an isometric isomorphism, thus reducing the problem to the case in Step 1 above.

We wish to point out that the use of spectral configurations can be avoided if one is only interested in the case $\left|\frac{1}{p}-\frac{1}{2}\right|>\left|\frac{1}{q}-\frac{1}{2}\right|$. Indeed, this situation can be entirely dealt with the maps $\gamma_{p, q}$ (see Proposition 4.1 or Theorem 4.14 for a similar argument, but in a different context). Also, the fact that we can assume representations to be non-degenerate (Step 2) is by no means obvious, and the paper GT16c grew out of our attempts to prove this.

1.1. Notation. We take $\mathbb{N}=\{1,2, \ldots\}$. For $n \in \mathbb{N}$ and $p \in[1, \infty)$, we write $\ell_{n}^{p}$ in place of $\ell^{p}(\{1, \ldots, n\})$, and we write $\ell^{p}$ in place of $\ell^{p}(\mathbb{Z})$. For a Banach space $E$, we write $\mathcal{B}(E)$ for the Banach algebra of bounded linear operators on $E$. For $p \in(1, \infty)$, we denote by $p^{\prime}$ its conjugate (Hölder) exponent, which is determined by the identity $\frac{1}{p}+\frac{1}{p^{\prime}}=1$. Consistently, for a Banach space $E$, we denote its dual space by $E^{\prime}$, and for a linear map $\pi: E \rightarrow F$ between Banach spaces $E$ and $F$, we denote by $\pi^{\prime}: F^{\prime} \rightarrow E^{\prime}$ its transpose map.

Locally compact groups are assumed to be Hausdorff, and will always be implicitly endowed with a (fixed) left Haar measure, which will be chosen to be the counting measure whenever the group is discrete. The left Haar measure of a locally compact group $G$ will be denoted by $\mu$, and we will denote by $\nu$ the right Haar measure on $G$ determined by $\nu(U)=\mu\left(U^{-1}\right)$ for all measurable sets $U \subseteq G$. The modular function of $G$ will be denoted by $\Delta: G \rightarrow \mathbb{R}_{+}$. We will repeatedly use the following identities:

$$
\int_{G} f(t) d \mu(t)=\int_{G} \Delta\left(t^{-1}\right) f\left(t^{-1}\right) d \mu(t)=\int_{G} f\left(t^{-1}\right) d \nu(t)=\int_{G} \Delta(t) f(t) d \nu(t),
$$

valid for all $f \in L^{1}(G, \mu)$.

\section{Preliminaries}

In this section, we recall and collect the necessary definitions and theorems that will be used throughout the paper. The only thing in this section that is really new is Proposition 2.3.

We begin by defining the main objects of study of this work.

Definition 2.1. Let $G$ be a locally compact group, and let $p \in[1, \infty)$. Denote by $\operatorname{Rep}_{p}(G)$ the class of all contractive representations of $L^{1}(G)$ on $L^{p}$-spaces. The full group $L^{p}$-operator algebra of $G$, denoted $F^{p}(G)$, is the completion of $L^{1}(G)$ in the norm given by

$$
\|f\|_{F^{p}(G)}=\sup \left\{\|\pi(f)\|: \pi \in \operatorname{Rep}_{p}(G)\right\}
$$

for $f \in L^{1}(G)$.

Denote by $\lambda_{p}: L^{1}(G) \rightarrow \mathcal{B}\left(L^{p}(G)\right)$ the left regular representation, which is given by $\lambda_{p}(f) \xi=f * \xi$ for $f \in L^{1}(G)$ and $\xi \in L^{p}(G)$. The algebra of p-pseudofunctions 
on $G$ (sometimes also called reduced group $L^{p}$-operator algebra of $G$ ), here denoted $F_{\lambda}^{p}(G)$, is the completion of $L^{1}(G)$ in the norm

$$
\|f\|_{F_{\lambda}^{p}(G)}=\left\|\lambda_{p}(f)\right\|_{\mathcal{B}\left(L^{p}(G)\right)}
$$

for $f \in L^{1}(G)$.

The algebra $F^{p}(G)$ has been defined in Phi13 and GT15b as the completion of $L^{1}(G)$ with respect to non-degenerate, contractive representations on $L^{p}$-spaces. In the proposition below, we show that this distinction is irrelevant. We recall the following, which is a particular case of a result from GT16c.

Theorem 2.2. (GT16c]). Let $A$ be a Banach algebra with a left contractive approximate identity, let $E$ be a reflexive Banach space, and let $\varphi: A \rightarrow \mathcal{B}(E)$ be a contractive homomorphism. Denote by $E_{0}$ the essential subspace of $\varphi$, this is, $E_{0}=\overline{\operatorname{span}} \varphi(A) E$. Then there exists a contractive idempotent $e \in \mathcal{B}(E)$ satisfying $e(E)=E_{0}$.

Proposition 2.3. Let $p \in[1, \infty)$ and let $G$ be a locally compact group. If $E$ is an $L^{p}$-space and $\pi: L^{1}(G) \rightarrow \mathcal{B}(E)$ is a contractive representation, then there exist an $L^{p}$-space $F$ and a contractive, non-degenerate representation $\varphi: L^{1}(G) \rightarrow \mathcal{B}(F)$ such that

$$
\|\pi(f)\| \leq\|\varphi(f)\|
$$

for all $f \in L^{1}(G)$. In particular, Definition 2.1 agrees with the definitions given in Phi13, and GT15b.

Proof. We treat the case $p=1$ first. In this case, we may take $\varphi$ to be the left regular representation $\lambda_{1}$. Indeed, let $\pi: L^{1}(G) \rightarrow \mathcal{B}(E)$ be a contractive representation on an $L^{1}$-space, and let $\left(a_{j}\right)_{j \in J}$ be a contractive approximate identity for $L^{1}(G)$. Then

$$
\|\pi(f)\| \leq\|f\|_{1}=\lim _{j \in J}\left\|f * a_{j}\right\|_{1}=\lim _{j \in J}\left\|\lambda_{1}(f) a_{j}\right\|_{1} \leq\left\|\lambda_{1}(f)\right\|,
$$

for all $f \in L^{1}(G)$, as desired.

Assume now that $p>1$. Let $\pi: L^{1}(G) \rightarrow \mathcal{B}(E)$ be a contractive representation on an $L^{p}$-space. Since $E$ is reflexive and $L^{1}(G)$ has a contractive approximate identity, it follows from Theorem 2.2 that there exists a contractive projection $e \in$ $\mathcal{B}(E)$ such that $e(E)$ is the essential subspace $E_{0}$ of $\pi$. Then $E_{0}$ is an $L^{p}$-space by Theorem 6 in Tza69. Let $\varphi: L^{1}(G) \rightarrow \mathcal{B}\left(E_{0}\right)$ be the restriction of $\pi$. Then $\varphi$ is a non-degenerate, contractive representation, and it is clear that $\|\pi(f)\|=\|\varphi(f)\|$ for all $f \in L^{1}(G)$.

The following duality principle was established in Proposition 2.18 of [GT15b].

Proposition 2.4. Let $G$ be a locally compact group, let $p \in(1, \infty)$, and let $p^{\prime}$ be its conjugate exponent. Then the inversion map on $G$ extends to a canonical isometric isomorphism $F^{p}(G) \cong F^{p^{\prime}}(G)$.

The next result is a combination of Theorem 2.30 and Corollary 3.20 in GT15b. The case $2 \leq q \leq p<\infty$ is obtained using duality, so we omit it.

Theorem 2.5. Let $G$ be a locally compact group. If $1 \leq p \leq q \leq 2$, then the identity map on $L^{1}(G)$ extends to a contractive homomorphism

$$
\gamma_{p, q}: F^{p}(G) \rightarrow F^{q}(G)
$$


with dense range. In particular, $\|f\|_{F^{q}(G)} \leq\|f\|_{F^{p}(G)}$ for every $f \in L^{1}(G)$.

If, moreover, $G$ is amenable, then:

(a) $\gamma_{p, q}$ is injective.

(b) $\gamma_{p, q}$ is surjective if and only if $G$ is finite.

Remark 2.6. When $p=2$, we have $F^{p}(G)=C^{*}(G)$, the full group $C^{*}$-algebra, and $F_{\lambda}^{p}(G)=C_{\lambda}^{*}(G)$, the reduced group $C^{*}$-algebra. In particular, when $G$ is abelian, then $\gamma_{p, 2}: F^{p}(G) \rightarrow C^{*}(G) \cong C_{0}(\widehat{G})$ is the Gelfand transform.

It follows from universality of the norm of $F^{p}(G)$ that the identity map on $L^{1}(G)$ extends to a contractive homomorphism $\kappa_{p}: F^{p}(G) \rightarrow F_{\lambda}^{p}(G)$ with dense range. The following is part of Theorem 3.7 in GT15b. This result has been independently obtained by Phillips, and will appear in Phi14.

Theorem 2.7. Let $G$ be a locally compact group, and let $p \in(1, \infty)$. The following are equivalent:

(1) The group $G$ is amenable.

(2) The canonical map $\kappa_{p}: F^{p}(G) \rightarrow F_{\lambda}^{p}(G)$ is an (isometric) isomorphism.

In view of the above result, we will identify $F^{p}(G)$ and $F_{\lambda}^{p}(G)$ in a canonical manner whenever $G$ is amenable.

\section{3. $F^{p}(\mathbb{Z})$ IS NOT ISOMORPHIC TO $F^{q}(\mathbb{Z})$}

The main step in the proof of Theorem 4.12 is to use the functoriality properties studied in GT14 to reduce the statement to the case where $G$ is a cyclic group (finite or infinite). While the case of a finite cyclic group will be dealt with using spectral configurations introduced in GT15a], the case of the infinite cyclic group has to be dealt with separately, and we do so in this section. Our goal here is to prove the following: for $p, q \in[1, \infty)$, there is an isometric isomorphism $F^{p}(\mathbb{Z}) \cong$ $F^{q}(\mathbb{Z})$ as Banach algebras if and only if $\left|\frac{1}{p}-\frac{1}{2}\right|=\left|\frac{1}{q}-\frac{1}{2}\right|$ (which is equivalent to $p$ and $q$ being either equal or Hölder conjugate); see Theorem 3.6. In order to prove this, we will reduce to the problem of ruling out the possibility of a canonical isomorphism for the cyclic group $\mathbb{Z}_{2}$. In this case, the norm of a certain element can be explicitly computed, yielding the result; see Proposition 3.3.

It should be pointed out that the methods of this section do not seem to generalize to other finite cyclic groups besides $\mathbb{Z}_{2}$. We will postpone dealing with such groups until the next section; see Theorem 4.12, and specifically the proof of Claim 2 in it. The approach we take here for the case of $\mathbb{Z}_{2}$ has the advantage of being completely elementary and it does not depend on results from other works (in particular, we do not need here anything about spectral configurations from GT15a).

We begin by looking at the group $L^{p}$-operator algebra of a finite cyclic group, which by amenability coincides with the algebra of $p$-pseudofunctions (this is the presentation we actually use). 
Example 3.1. Let $n \in \mathbb{N}$ and let $p \in[1, \infty)$. Then $L^{p}$-group algebra $F^{p}\left(\mathbb{Z}_{n}\right)$ is the Banach subalgebra of $\mathcal{B}\left(\ell_{n}^{p}\right)$ generated by the cyclic shift $s_{n}$ of order $n$

$$
s_{n}=\left(\begin{array}{ccccc}
0 & & & & 1 \\
1 & 0 & & & \\
& \ddots & \ddots & & \\
& & \ddots & 0 & \\
& & & 1 & 0
\end{array}\right) .
$$

(The algebra $\mathcal{B}\left(\ell_{n}^{p}\right)$ is just $M_{n}$ with the $L^{p}$-operator norm.) It is easy to check that $F^{p}\left(\mathbb{Z}_{n}\right)$ is algebraically isomorphic to $\mathbb{C}^{n}$, but the canonical embedding $F^{p}\left(\mathbb{Z}_{n}\right) \rightarrow$ $M_{n}$ is not as diagonal matrices. In fact, computing the norm of an element in $F^{p}\left(\mathbb{Z}_{n}\right)$ is challenging for $p$ other than from 1 and 2 , essentially because computing $p$-norms of matrices that are not diagonal is difficult. Indeed, let $\omega_{n}=e^{\frac{2 \pi i}{n}}$, and set

$$
u_{n}=\frac{1}{\sqrt{n}}\left(\begin{array}{ccccc}
1 & 1 & 1 & \cdots & 1 \\
1 & \omega_{n} & \omega_{n}^{2} & \cdots & \omega_{n}^{n-1} \\
1 & \omega_{n}^{2} & \omega_{n}^{4} & \cdots & \omega_{n}^{2(n-1)} \\
\vdots & \vdots & \vdots & \ddots & \vdots \\
1 & \omega_{n}^{n-1} & \omega_{n}^{2(n-1)} & \cdots & \omega_{n}^{(n-1)^{2}}
\end{array}\right) .
$$

If $\xi=\left(\xi_{0}, \ldots, \xi_{n-1}\right) \in \mathbb{C}^{n} \cong F^{p}\left(\mathbb{Z}_{n}\right)$, then its norm in $F^{p}\left(\mathbb{Z}_{n}\right)$ is

$$
\|\xi\|_{F^{p}\left(\mathbb{Z}_{n}\right)}=\left\|u_{n}\left(\begin{array}{cccc}
\xi_{0} & & & \\
& \xi_{1} & & \\
& & \ddots & \\
& & & \xi_{n-1}
\end{array}\right) u_{n}^{-1}\right\|_{\mathcal{B}\left(\ell_{n}^{p}\right)} .
$$

The matrix $u_{n}$ is unitary (its inverse is the transpose of its conjugate), and hence $\|\xi\|_{F^{2}\left(\mathbb{Z}_{n}\right)}=\|\xi\|_{\infty}$. Moreover, if $1 \leq p \leq q \leq 2$, then $\|\cdot\|_{F^{q}\left(\mathbb{Z}_{n}\right)} \leq\|\cdot\|_{F^{p}\left(\mathbb{Z}_{n}\right)}$ by Theorem 2.5. In particular, the norm $\|\cdot\|_{F^{p}\left(\mathbb{Z}_{n}\right)}$ always dominates the norm $\|\cdot\|_{\infty}$.

The automorphism group of $F^{p}\left(\mathbb{Z}_{n}\right)$ is not easy to describe when $p \neq 2$, since not every permutation of the coordinates of $\mathbb{C}^{n}$ is isometric. The next proposition asserts that the cyclic shift on $\mathbb{C}^{n}$ is isometric.

Proposition 3.2. Let $n \in \mathbb{N}$ and let $p \in[1, \infty)$. Denote by $\tau: \mathbb{C}^{n} \rightarrow \mathbb{C}^{n}$ the cyclic forward shift, this is,

$$
\tau\left(\xi_{0}, \ldots, \xi_{n-1}\right)=\left(\xi_{n-1}, \xi_{0}, \ldots, \xi_{n-2}\right)
$$

for all $\left(\xi_{0}, \ldots, \xi_{n-1}\right) \in \mathbb{C}^{n}$. Then $\tau$ induces an isometric isomorphism $F^{p}\left(\mathbb{Z}_{n}\right) \rightarrow$ $F^{p}\left(\mathbb{Z}_{n}\right)$.

Proof. We follow the notation from Example 3.1. except that we write $u$ in place of $u_{n}$, and we write $s$ in place of $s_{n}$.

For $\xi \in \mathbb{C}^{n}$, let $d(\xi)$ denote the diagonal $n \times n$ matrix with $d(\xi)_{j, j}=\xi_{j}$ for $j=0, \ldots, n-1$. Denote by $\rho: \mathbb{C}^{n} \rightarrow M_{n}$ the unital homomorphism given by $\rho(\xi)=u d(\xi) u^{-1}$ for $\xi \in \mathbb{C}^{n}$. Then

$$
\|\xi\|_{F^{p}\left(\mathbb{Z}_{n}\right)}=\|\rho(\xi)\|_{\mathcal{B}\left(\ell_{n}^{p}\right)}=\left\|u d(\xi) u^{-1}\right\|_{\mathcal{B}\left(\ell_{n}^{p}\right)}
$$


for all $\xi \in \mathbb{C}^{n}$.

Set $\omega=\left(1, \omega_{n}^{1}, \ldots, \omega_{n}^{n-1}\right) \in \mathbb{C}^{n}$, and denote by $\bar{\omega}$ its (coordinate-wise) conjugate. Given $\xi \in \mathbb{C}^{n}$, one checks that

$$
\mathrm{d}(\tau(\xi))=s d(\xi) s^{-1}, \quad u s=d(\omega) u, \quad \text { and } \quad s^{-1} u^{-1}=u^{-1} d(\bar{\omega}) .
$$

It follows that

$$
\begin{aligned}
\|\tau(\xi)\|_{F^{p}\left(\mathbb{Z}_{n}\right)} & =\left\|u \mathrm{~d}(\tau(\xi)) u^{-1}\right\|_{\mathcal{B}\left(\ell_{n}^{p}\right)} \\
& =\left\|u s d(\xi) s^{-1} u^{-1}\right\|_{\mathcal{B}\left(\ell_{n}^{p}\right)} \\
& =\left\|d(\omega) u d(\xi) u^{-1} d(\bar{\omega})\right\|_{\mathcal{B}\left(\ell_{n}^{p}\right)} .
\end{aligned}
$$

Since $d(\omega)$ and $d(\bar{\omega})$ are isometries of $\ell_{n}^{p}$, we conclude that

$$
\|\tau(\xi)\|_{F^{p}\left(\mathbb{Z}_{n}\right)}=\left\|d(\omega) u \tau(\xi) u^{-1} d(\bar{\omega})\right\|_{\mathcal{B}\left(\ell_{n}^{p}\right)}=\left\|u d(\xi) u^{-1}\right\|_{\mathcal{B}\left(\ell_{n}^{p}\right)}=\|\xi\|_{F^{p}\left(\mathbb{Z}_{n}\right)},
$$

as desired.

The fact that $F^{p}\left(\mathbb{Z}_{2}\right)$ is isometrically isomorphic to $F^{q}\left(\mathbb{Z}_{2}\right)$ only in the "obvious" cases can be proved directly by computing the norm of a special element, as we show below.

Proposition 3.3. Let $p, q \in[1, \infty)$. Then $F^{p}\left(\mathbb{Z}_{2}\right)$ is isometrically isomorphic, as Banach algebras, to $F^{q}\left(\mathbb{Z}_{2}\right)$ if and only if $\left|\frac{1}{p}-\frac{1}{2}\right|=\left|\frac{1}{q}-\frac{1}{2}\right|$.

Proof. The "if" implication follows from Proposition 2.4. We proceed to show the "only if" implication. Given $t \in[1, \infty)$, we claim that

$$
\|(1, i)\|_{F^{t}\left(\mathbb{Z}_{2}\right)}=2^{\left|\frac{1}{t}-\frac{1}{2}\right|} .
$$

By Proposition 2.4 the quantity on the left-hand side remains unchanged if one replaces $t$ with its conjugate exponent. Since the same holds for the quantity on the right-hand side, it follows that it is enough to prove the claim for $t \in[1,2]$.

Define a continuous function $\delta:[1,2] \rightarrow \mathbb{R}$ by $\delta(t)=\|(1, i)\|_{F^{t}\left(\mathbb{Z}_{2}\right)}$ for $t \in[1,2]$. Let $a$ be the matrix

$$
a=\frac{1}{2}\left(\begin{array}{cc}
1+i & 1-i \\
1-i & 1+i
\end{array}\right) .
$$

Then $\delta(t)=\|a\|_{\mathcal{B}\left(\ell_{2}^{t}\right)}$ for all $t \in[1,2]$. The values of $\delta$ at $t=1$ and $t=2$ are easy to compute, and we have $\delta(1)=2^{\frac{1}{2}}$ and $\delta(2)=1$. Fix $t \in(1,2)$ and let $\theta \in(0,1)$ satisfy

$$
\frac{1}{t}=\frac{1-\theta}{1}+\frac{\theta}{2}
$$

Using the Riesz-Thorin Interpolation Theorem in [1,2], we conclude that

$$
\delta(t) \leq \delta(1)^{1-\theta} \cdot \delta(2)^{\theta}=2^{\frac{1}{2}\left(\frac{2}{t}-1\right)} \cdot 1=2^{\frac{1}{t}-\frac{1}{2}} .
$$

For the converse inequality, fix $t \in[1,2]$ and consider the vector $\xi=\left(\begin{array}{l}1 \\ 0\end{array}\right) \in \ell_{2}^{t}$. Then $\|\xi\|_{t}=1$ and $a x=\frac{1}{2}\left(\begin{array}{c}1+i \\ 1-i\end{array}\right)$. We have

$$
\left\|\frac{1}{2}\left(\begin{array}{c}
1+i \\
1-i
\end{array}\right)\right\|_{t}=\frac{1}{2}\left(|1+i|^{t}+|1-i|^{t}\right)^{\frac{1}{t}}=2^{\left(\frac{1}{t}-\frac{1}{2}\right)} .
$$

We conclude that

$$
\delta(t)=\|a\|_{\mathcal{B}\left(\ell_{2}^{t}\right)} \geq \frac{\|a x\|_{t}}{\|\xi\|_{t}}=2^{\left(\frac{1}{t}-\frac{1}{2}\right)} .
$$

This shows that $\delta(t)=2^{\left(\frac{1}{t}-\frac{1}{2}\right)}$ for all $t \in[1,2]$, and the claim is proved. 
Now let $p, q \in[1, \infty)$ and let $\psi: F^{p}\left(\mathbb{Z}_{2}\right) \rightarrow F^{q}\left(\mathbb{Z}_{2}\right)$ be an isometric isomorphism. Since $\psi$ is an algebra isomorphism, we must have either $\psi(x, y)=(x, y)$ or $\psi(x, y)=$ $(y, x)$ for all $(x, y) \in \mathbb{C}^{2}$. By Proposition 3.2, the flip $(x, y) \mapsto(y, x)$ is an isometric isomorphism of $F^{q}\left(\mathbb{Z}_{2}\right)$, so we may assume that $\psi$ is the identity map on $\mathbb{C}^{2}$. It follows that $\|(1, i)\|_{F^{p}\left(\mathbb{Z}_{2}\right)}=\|(1, i)\|_{F^{q}\left(\mathbb{Z}_{2}\right)}$, so $\left|\frac{1}{p}-\frac{1}{2}\right|=\left|\frac{1}{q}-\frac{1}{2}\right|$ and the proof is complete.

Remark 3.4. Adopt the notation from the proof above. Then the function $\delta$ attains the upper bound given by the Riesz-Thorin Interpolation Theorem, which is a rare situation. This fortunate coincidence makes the argument possible, but it is not clear to us how to generalize these computations to other cyclic groups, or even to $\mathbb{Z}_{3}$. However, knowing the result just for $\mathbb{Z}_{2}$ is enough to prove Theorem 3.6.

The following is probably standard, but we have not been able to find a reference in the literature. Accordingly, we prove it here.

Proposition 3.5. Let $f: S^{1} \rightarrow S^{1}$ be a homeomorphism. Then there exists $\zeta \in S^{1}$ such that $f(-\zeta)=-f(\zeta)$.

Proof. Regard $f$ as a homeomorphism $h:[0,2 \pi] \rightarrow[0,2 \pi]$ with either $h(0)=0$ (if $f$ is orientation preserving) or $h(0)=2 \pi$ (if $f$ is orientation reversing). Without loss of generality, assume that $h(0)=0$, and hence that $h$ is strictly increasing with $h(2 \pi)=2 \pi$. Let $g:[0, \pi] \rightarrow \mathbb{R}$ be given by

$$
g(t)=\frac{h(t+\pi)-h(t)}{\pi}
$$

for $t \in[0, \pi]$. We need to find $s \in[0, \pi]$ with $g(s)=1$. If $h(\pi)=\pi$, then $g(\pi)=1$ and we are done. If $h(\pi)>\pi$, we have $g(0)>1$, while $g(\pi)<1$. Likewise, if $h(\pi)<\pi$, then $g(0)<1$ and $g(\pi)>1$. In either case, the conclusion then follows from the Mean Value Theorem.

We are now ready to prove that for $p, q \in[1, \infty)$, the algebras $F^{p}(\mathbb{Z})$ and $F^{q}(\mathbb{Z})$ are (abstractly) isometrically isomorphic only in the trivial case when $\left|\frac{1}{p}-\frac{1}{2}\right|=$ $\left|\frac{1}{q}-\frac{1}{2}\right|$. This result should be compared with part (2) of Corollary 3.20 in [GT15b], here reproduced as Theorem 2.5, where only the canonical homomorphism is considered. The strategy will be to use Proposition 3.5 to reduce to the case when the group is $\mathbb{Z}_{2}$, which is Proposition 3.3. The fact that the spectrum of $F^{p}(\mathbb{Z})$ is the circle is crucial in our proof, and we do not know how to generalize these methods to directly deal with, for example, $\mathbb{Z}^{2}$.

Theorem 3.6. Let $p, q \in[1, \infty)$. Then $F^{p}(\mathbb{Z})$ and $F^{q}(\mathbb{Z})$ are isometrically isomorphic (as Banach algebras) if and only if $\left|\frac{1}{p}-\frac{1}{2}\right|=\left|\frac{1}{q}-\frac{1}{2}\right|$.

Proof. The "if" implication follows from Proposition 2.4. Let us show the converse.

By Proposition 3.13 in GT15b], the maximal ideal spaces of $F^{p}(\mathbb{Z})$ and $F^{q}(\mathbb{Z})$ are canonically homeomorphic to $S^{1}$. We let $\Gamma_{p}: F^{p}(\mathbb{Z}) \rightarrow C\left(S^{1}\right)$ denote the Gelfand transform, which maps the canonical generating invertible isometry $u \in F^{p}(\mathbb{Z})$, associated to $1 \in \mathbb{Z}$, to the canonical inclusion $\iota: S^{1} \rightarrow \mathbb{C}$.

Let $\varphi: F^{p}(\mathbb{Z}) \rightarrow F^{q}(\mathbb{Z})$ be an isometric isomorphism. Then $\varphi$ induces a homeomorphism $f: S^{1} \rightarrow S^{1}$ that maps $z \in S^{1}$ to the unique point $f(z) \in S^{1}$ satisfying $\operatorname{ev}_{z} \circ \varphi=\operatorname{ev}_{f(z)}: F^{p}(\mathbb{Z}) \rightarrow \mathbb{C}$. 
Use Proposition 3.5 to choose $\zeta_{0} \in S^{1}$ such that $f\left(-\zeta_{0}\right)=-f\left(\zeta_{0}\right)$. Denote by $\pi_{p}: F^{p}(\mathbb{Z}) \rightarrow F^{p}\left(\mathbb{Z}_{2}\right)$ and $\pi_{q}: F^{p}(\mathbb{Z}) \rightarrow F^{q}\left(\mathbb{Z}_{2}\right)$ the canonical homomorphisms associated with the surjective map $\mathbb{Z} \rightarrow \mathbb{Z}_{2}$, which are quotient maps by Theorem 2.5 in GT14. Let $\omega_{\zeta_{0}}: F^{p}(\mathbb{Z}) \rightarrow F^{p}(\mathbb{Z})$ be the isometric isomorphism induced by multiplying the canonical generator $u \in F^{p}(\mathbb{Z})$ by $\zeta_{0}$. Analogously, let $\omega_{f\left(\zeta_{0}\right)}: F^{q}(\mathbb{Z}) \rightarrow F^{q}(\mathbb{Z})$ be the isometric isomorphism induced by multiplying $u \in F^{q}(\mathbb{Z})$ by $f\left(\zeta_{0}\right)$. Then the following diagram commutes:

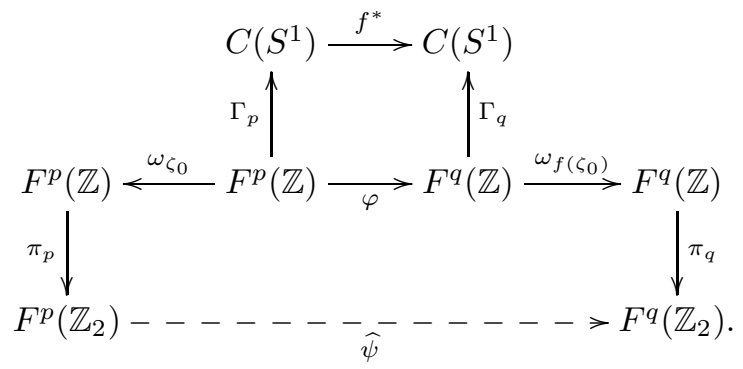

Define an isometric isomorphism $\psi: F^{p}(\mathbb{Z}) \rightarrow F^{q}(\mathbb{Z})$ by $\psi=\omega_{f\left(\zeta_{0}\right)} \circ \varphi \circ \omega_{\zeta_{0}}^{-1}$. One checks that $\psi\left(\operatorname{ker}\left(\pi_{p}\right)\right)=\operatorname{ker}\left(\pi_{q}\right)$. It follows that $\psi$ induces an isometric isomorphism $\widehat{\psi}: F^{p}\left(\mathbb{Z}_{2}\right) \rightarrow F^{q}\left(\mathbb{Z}_{2}\right)$. By Proposition 3.3, this implies that $\left|\frac{1}{p}-\frac{1}{2}\right|=$ $\left|\frac{1}{q}-\frac{1}{2}\right|$, as desired.

\section{Nonrepresentability ON $L^{q}$-SPACES}

The goal of this section is to show that for a nontrivial locally compact group $G$ and for $p, q \in[1, \infty)$ distinct, the Banach algebras $F^{p}(G), F_{\lambda}^{p}(G), P M_{p}(G)$ and $C V_{p}(G)$ can be represented on an $L^{q}$-space only in the trivial cases, namely if either $p=2$ and $G$ is abelian; or if $p$ and $q$ are Hölder conjugate; see Theorem 4.12, The main step in the proof is to use the functoriality properties of these objects, studied in GT14, to reduce the statement to the case where $G$ is a cyclic group (finite or infinite). The case of a finite cyclic group will be dealt with using spectral configurations as in GT15a, as well as the canonical maps $\gamma_{p, q}: F^{p}(G) \rightarrow F^{q}(G)$ constructed in Theorem 3.7 in GT15b.

Proposition 4.1 below is our first preparatory result on representability of full group $L^{p}$-operator algebras on $L^{q}$-spaces. We need some notation first. Let $G$ be a locally compact group, and denote by $\Delta: G \rightarrow \mathbb{R}$ its modular function. For $f \in L^{1}(G)$, let $f^{\sharp}: G \rightarrow \mathbb{C}$ be given by $f^{\sharp}(s)=\Delta\left(s^{-1}\right) f\left(s^{-1}\right)$ for all $s \in G$. It is easy to check that the map $\sharp: L^{1}(G) \rightarrow L^{1}(G)$ is an anti-multiplicative isometric linear map of order two.

Proposition 4.1. Let $G$ be a locally compact group, and let $p, q \in[1, \infty)$ satisfy $\left|\frac{1}{p}-\frac{1}{2}\right|>\left|\frac{1}{q}-\frac{1}{2}\right|$. Suppose that $F^{p}(G)$ is isometrically representable on an $L^{q}-$ space.

(1) If $p, q \in[1,2]$ or $p, q \in[2, \infty)$, then the identity map on $L^{1}(G)$ extends to an isometric isomorphism $F^{p}(G) \cong F^{q}(G)$.

(2) If $p \in[1,2]$ and $q \in[2, \infty)$, or if $q \in[1,2]$ and $p \in[2, \infty)$, then the map $\sharp$ on $L^{1}(G)$ induces, when composed with the transpose map $\mathcal{B}\left(L^{p}(G)\right) \rightarrow$ $\mathcal{B}\left(L^{p^{\prime}}(G)\right)$, an isometric isomorphism $F^{p}(G) \cong F^{q}(G)$. 
Proof. (1). The result is trivial when $p=q$. Suppose first that $1 \leq p<q \leq 2$. Suppose that there exist an $L^{q}$-space $E$ and an isometric representation $\varphi: F^{p}(G) \rightarrow$ $\mathcal{B}(E)$. Let $\iota_{p}: L^{1}(G) \rightarrow F^{p}(G)$ be the canonical contractive inclusion with dense range. Then

$$
\psi=\varphi \circ \iota_{p}: L^{1}(G) \rightarrow \mathcal{B}(E)
$$

is a contractive representation of $L^{1}(G)$ on an $L^{q}$-space.

Let $f \in L^{1}(G)$. Using Theorem 2.5 at the first step, we deduce that

$$
\|f\|_{F^{q}(G)} \leq\|f\|_{F^{p}(G)}=\|\psi(f)\|_{\mathcal{B}(F)} \leq\|f\|_{F^{q}(G)} .
$$

Hence $\|f\|_{F^{q}(G)}=\|f\|_{F^{p}(G)}$. Since $f \in L^{1}(G)$ is arbitrary, it follows that the identity on $L^{1}(G)$ extends to an isometric isomorphism $F^{p}(G) \rightarrow F^{q}(G)$, as desired.

The case $2 \leq q<p<\infty$ follows from duality; see Proposition 2.4.

(2). We can assume, without loss of generality, that $1 \leq p \leq 2 \leq q<\infty$. Denote by $q^{\prime} \in[1,2]$ the Hölder conjugate exponent of $q$. By Proposition 2.4 the map $\sharp: L^{1}(G) \rightarrow L^{1}(G)$, when composed with the transpose map $\mathcal{B}\left(L^{q}(G)\right) \rightarrow$ $\mathcal{B}\left(L^{q^{\prime}}(G)\right)$, extends to an isometric isomorphism $F^{q}(G) \rightarrow F^{q^{\prime}}(G)$. (The details are in the proof of Lemma 2.16 of GT15b, the main point being that given a representation of $G$ on an $L^{q}$-space, its dual representation is a representation of $G^{\text {op }}$ on $L^{q^{\prime}}$ which induces the same norm on $L^{1}(G)$. One composes this isometric antiisomorphism with the map $\sharp$ to get a multiplicative (isometric) isomorphism.) Since the identity map on $L^{1}(G)$ extends to an isometric isomorphism $F^{p}(G) \rightarrow F^{q^{\prime}}(G)$ by part (1), the result follows.

Besides $F^{p}(G)$ and $F_{\lambda}^{p}(G)$, the other two Banach algebras we will be concerned with, at least when $p>1$, are the algebra $P M_{p}(G)$ of $p$-pseudomeasures, and the algebra $C V_{p}(G)$ of $p$-convolvers. These are, respectively, the ultraweak closure, and the bicommutant, of $F_{\lambda}^{p}(G)$ in $\mathcal{B}\left(L^{p}(G)\right)$.

Algebras of pseudomeasures and of convolvers on groups have been thoroughly studied since their inception by Herz in the early 70's; see Her73. It is clear that $P M_{p}(G) \subseteq C V_{p}(G)$, and it is conjectured that they are equal for every locally compact group $G$ and every Hölder exponent $p \in(1, \infty)$. The conjecture is known to be true if $p=2$, or if $G$ is amenable ( approximation property (Cow98).

Our next goal is showing that these convolution algebras are never operator algebras when $p \neq 2$. We first need two results about $C^{*}$-algebras which are interesting in their own right. The first one is well-known, and it follows, from example, from Theorem 10 of Bon54.

Theorem 4.2. Let $A$ be a $C^{*}$-algebra, let $B$ be a Banach algebra, and let $\varphi: A \rightarrow B$ be a contractive, injective homomorphism. Then $\varphi$ is isometric.

Remark 4.3. If in the theorem above $\varphi$ is not assumed to be injective, then the conclusion is that it is a quotient map. On the other hand, we must assume that $\varphi$ is contractive, and not merely continuous, for the result to hold; counterexamples are easy to construct. The result also fails for not necessarily self-adjoint operator algebras, even for uniform algebras.

The next fact about $C^{*}$-algebras is proved in [GT16c], and had not been noticed before, at least not in this generality. The main difficulty is dealing with nondegenerate representations, for which Theorem 2.2 is essential. 
Theorem 4.4. (GT16c] Let $A$ be a $C^{*}$-algebra. Then the following are equivalent:

(1) A can be isometrically represented on an $L^{p}$-space, for some $p \in[1, \infty) \backslash\{2\}$;

(2) $A$ can be isometrically represented on an $L^{p}$-space, for all $p \in[1, \infty)$;

(3) $A$ is commutative (and hence $A \cong C_{0}(X)$ for $X=\operatorname{Max}(A)$ ).

We also need to recall the following, whose proof can be found, for example, in [GT14. For a Banach algebra $A$, we denote its left (respectively, right, twosided) multiplier algebra by $M_{L}(A)$ (respectively, $M_{R}(A)$ and $M(A)$ ), and we write $\iota_{L}: A \rightarrow M_{L}(A)$ (respectively, $\iota_{R}: A \rightarrow M_{R}(A)$ and $\iota: A \rightarrow M(A)$ ) for the canonical inclusion. When confusion may arise, we write the product in $M(A)$ with a dot.

Theorem 4.5. Let $A$ be a Banach algebra with a left (respectively, right, two-sided) contractive approximate identity. Let $X$ be a Banach space and let $\varphi: A \rightarrow \mathcal{B}(X)$ be a non-degenerate contractive representation. Then there exists a unique unital contractive homomorphism $\psi_{L}: M_{L}(A) \rightarrow \mathcal{B}(X)$ (respectively, $\psi_{R}: M_{R}(A) \rightarrow \mathcal{B}(X)$ and $\psi: M(A) \rightarrow \mathcal{B}(X)$ ) satisfying $\psi_{L} \circ \iota_{L}=\varphi$ (respectively, $\psi_{R} \circ \iota_{R}=\varphi$ and $\psi \circ \iota=\varphi)$. Moreover, the map $\psi_{L}$ is given by

$$
\psi_{L}(m)(\varphi(a)(\xi))=\varphi(m \cdot a)(\xi)
$$

for all $m \in M_{L}(A)$, for all $a \in A$ and for all $\xi \in X$.

The following theorem generalizes a result of Neufang and Runde, Theorem 2.2 in [NR09, where the authors assumed that the group in question was amenable and had a closed infinite abelian subgroup. Observe that for $G=\mathbb{Z}$, Theorem 4.6 follows from the main result of [GT16a], since there it is shown that for $p \neq 2$, there exists a semisimple quotient $A_{V}$ of $F^{p}(\mathbb{Z})$ that is not representable on $L^{q}$ for any $1 \leq q<\infty$. Indeed, if $F^{p}(\mathbb{Z})$ were an operator algebra, so would be any of its quotients. Recall that a unital commutative, semisimple operator algebra is isometrically isomorphic to a closed subalgebra of $C(X)$ for a compact Hausdorff space $X$ (that is, it is a uniform algebra). Hence, if $F^{p}(\mathbb{Z})$ were an operator algebra, then $A_{V}$ would be a uniform algebra, hence isometrically representable on an $L^{q_{-}}$ space for any $q \in[1, \infty)$.

Theorem 4.6 will be used in the proof of Theorem 4.12, which gives a much more general result. In the proof below, for a locally compact group $G$ and $p \in[1, \infty)$, and to emphasize the role played by $G$, we will denote by $\gamma_{p, 2}^{G}: F^{p}(G) \rightarrow C^{*}(G)$ the map from Theorem 2.5.

Theorem 4.6. Let $G$ be a locally compact group and let $p \in[1, \infty)$. Then one of $F^{p}(G), F_{\lambda}^{p}(G), P M_{p}(G)$ or $C V_{p}(G)$ is an operator algebra if and only if either $p=2$ or $G$ is the trivial group.

Proof. The "if" implication is obvious if $G$ is the trivial group, since the associated Banach algebras are all $\mathbb{C}$, while the statement is clear if $p=2$.

For the "only if" implication, it is clear that if either $P M_{p}(G)$ or $C V_{p}(G)$ is an operator algebra, then so is $F_{\lambda}^{p}(G)$, since there are isometric inclusions

$$
F_{\lambda}^{p}(G) \subseteq P M_{p}(G) \subseteq C V_{p}(G) .
$$

Assume now that $F^{p}(G)$ is an operator algebra and that $p \neq 2$. There is a canonical identification of $F^{p}(G)$ with $C^{*}(G)$, in view of Proposition 4.1 and Remark 2.6 Let $\kappa_{p}: F^{p}(G)=C^{*}(G) \rightarrow F_{\lambda}^{p}(G)$ denote the canonical contractive homomorphism 
with dense range. It is well-known that the quotient $C^{*}(G) / \operatorname{ker}\left(\kappa_{p}\right)$ is a $C^{*}$-algebra. The induced map

$$
\widehat{\kappa_{p}}: C^{*}(G) / \operatorname{ker}\left(\kappa_{p}\right) \rightarrow F_{\lambda}^{p}(G)
$$

is an injective, contractive homomorphism. Now, Theorem 4.2 shows that $\widehat{\kappa_{p}}$ is isometric. Since $\kappa_{p}$, and hence $\widehat{\kappa}_{p}$, has dense range, it follows that $F_{\lambda}^{p}(G)$ is isometrically isomorphic to $C^{*}(G) / \operatorname{ker}\left(\kappa_{p}\right)$. In particular, $F_{\lambda}^{p}(G)$ is a $C^{*}$-algebra, and hence an operator algebra itself.

It is therefore enough to show the statement assuming that $F_{\lambda}^{p}(G)$ is an operator algebra. Let $\mathcal{H}$ be a Hilbert space and let $\varphi: F_{\lambda}^{p}(G) \rightarrow \mathcal{B}(\mathcal{H})$ be an isometric representation.

Claim: $F_{\lambda}^{p}(G)$ is a $C^{*}$-algebra. Note that $A$ has a (two-sided) contractive approximate identity, since so does $L^{1}(G)$ and there is a contractive homomorphism $\iota: L^{1}(G) \rightarrow F_{\lambda}^{p}(G)$ with dense range. Moreover, since

$$
\overline{\left\{\varphi(a) \eta: a \in F_{\lambda}^{p}(G), \eta \in \mathcal{H}\right\}}
$$

is itself a Hilbert space, we may assume that the representation $\varphi$ is non-degenerate. It follows from Theorem 4.5 that the algebra $M\left(F_{\lambda}^{p}(G)\right) \subseteq \mathcal{B}\left(F_{\lambda}^{p}(G)\right)$ of multipliers on $F_{\lambda}^{p}(G)$, is unitally representable on $\mathcal{H}$.

By Corollary 2.5 in GT16b], there is a canonical isometric identification of the multiplier algebra $M\left(F_{\lambda}^{p}(G)\right) \subseteq \mathcal{B}\left(F_{\lambda}^{p}(G)\right)$, with the Banach algebra

$$
C\left(F_{\lambda}^{p}(G)\right)=\left\{x \in \mathcal{B}\left(L^{p}(G)\right): x a, a x \in F_{\lambda}^{p}(G) \text { for all } a \in F_{\lambda}^{p}(G)\right\} \subseteq \mathcal{B}\left(L^{p}(G)\right)
$$

of centralizers of $F_{\lambda}^{p}(G)$. Denote by $\psi: C\left(F_{\lambda}^{p}(G)\right) \rightarrow \mathcal{B}(\mathcal{H})$ the resulting unital, isometric representation.

There is an obvious identification of $G$ with a subgroup of the invertible isometries of $C\left(F_{\lambda}^{p}(G)\right)$, given by letting a group element $g \in G$ act on $L^{p}(G)$ as the convolution operator with respect to the point mass measure $\delta_{g}$. Now, for $g \in G$, set $u_{g}=\psi\left(\delta_{g}\right)$. Then $u_{g}$ is an invertible isometry on $\mathcal{H}$, that is, a unitary operator. Moreover, the map $u: G \rightarrow \mathcal{U}(\mathcal{H})$, given by $g \mapsto u_{g}$, is easily seen to be a strongly-continuous unitary representation of $G$ on $\mathcal{H}$. The integrated form $\rho_{u}: L^{1}(G) \rightarrow \mathcal{B}(\mathcal{H})$ of $u$ is therefore a contractive, non-degenerate *-homomorphism. Whence the subalgebra $\rho_{u}\left(L^{1}(G)\right) \subseteq \mathcal{B}(\mathcal{H})$ is closed under the adjoint operation. Moreover, it is clear that the following diagram commutes:

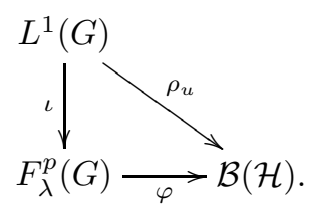

We conclude that $\varphi\left(F_{\lambda}^{p}(G)\right)$, which equals $\overline{\rho_{u}\left(L^{1}(G)\right)}$, is a closed $*$-subalgebra of $\mathcal{B}(\mathcal{H})$, that is, a $C^{*}$-algebra. The claim is proved.

It follows from Theorem 4.4 that $F_{\lambda}^{p}(G)$ is commutative. Thus $G$ is itself commutative, and in particular $F_{\lambda}^{q}(G)=F^{q}(G)$ for all $q \in[1, \infty)$, by Theorem 3.7 in GT15b.

The map $\gamma_{p, 2}^{G}: F_{\lambda}^{p}(G) \rightarrow C_{\lambda}^{*}(G)$ is an isometric isomorphism by Proposition 4.1. The fact that $\gamma_{p, 2}^{G}$ is surjective implies that $G$ is finite, by Theorem 2.5. Using that $\gamma_{p, 2}^{G}$ is isometric, we will show that $G$ must be the trivial group.

Using finiteness of $G$, let $g \in G$ be an element with maximum order. Set $n=\operatorname{ord}(g) \geq 1$, and let $j: \mathbb{Z}_{n} \hookrightarrow G$ be the group homomorphism determined 
by $j(1)=g$. By Proposition 2.3 in [GT14, there are natural isometric embeddings $j_{p}: F_{\lambda}^{p}\left(\mathbb{Z}_{n}\right) \hookrightarrow F_{\lambda}^{p}(G)$ and $j_{2}: C_{\lambda}^{*}\left(\mathbb{Z}_{n}\right) \hookrightarrow C_{\lambda}^{*}(G)$. Naturality of the maps involved implies that the following diagram is commutative:

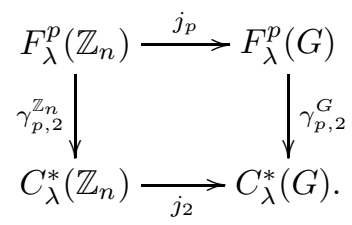

In particular, $\gamma_{p, 2}^{\mathbb{Z}_{n}}: F_{\lambda}^{p}\left(\mathbb{Z}_{n}\right) \rightarrow C_{\lambda}^{*}\left(\mathbb{Z}_{n}\right)$ is an isometric isomorphism. (This map is really just the identity on $\mathbb{C}^{n}$.)

Set $\omega=e^{\frac{2 \pi i}{n}} \in S^{1}$. Using that $p \neq 2$ together with Proposition 2.8 in GT15a (see also the comments above it), we conclude that if $x \in F_{\lambda}^{p}\left(\mathbb{Z}_{n}\right)$ is an invertible isometry, then there exist $\zeta \in S^{1}$ and $k \in\{0, \ldots, n-1\}$ such that, under the algebraic identification of $F_{\lambda}^{p}\left(\mathbb{Z}_{n}\right)$ with $\mathbb{C}^{n}$, we have

$$
x=\left(\zeta, \zeta \omega^{k}, \ldots, \zeta \omega^{k(n-1)}\right) .
$$

In particular, if $n>1$, then not every element in $\left(S^{1}\right)^{n} \subseteq \mathbb{C}^{n}$ has norm one in $F_{\lambda}^{p}\left(\mathbb{Z}_{n}\right)$. Since this certainly is the case in $C_{\lambda}^{*}\left(\mathbb{Z}_{n}\right)$, we must have $n=1$. By the choice of $n$, we conclude that $G$ must be the trivial group, and the proof of the theorem is finished.

In contrast with Theorem 4.6 we point out that some $L^{p}$-operator group algebras are contractively and isomorphically representable on Hilbert spaces. For example, for any finite group $G$, abelian or not, and for any $p \in[1, \infty)$, the map $\gamma_{p, 2}: F^{p}(G) \rightarrow C^{*}(G)$ from Theorem 2.5 is a contractive isomorphism.

For a locally compact group $G$, we review the definitions of the Banach algebras $M^{p}(G)$ and $M_{\lambda}^{p}(G)$ from GT14. Recall that $M^{1}(G)$ is the (unital) Banach algebra of finite complex Radon measures on $G$, and it can be identified with the multiplier algebra of $L^{1}(G)$. In particular, observe that the left regular representation $\lambda_{p}: L^{1}(G) \rightarrow \mathcal{B}\left(L^{p}(G)\right)$ can be extended to a representation $\lambda_{p}: M^{1}(G) \rightarrow \mathcal{B}\left(L^{p}(G)\right)$.

Definition 4.7. Let $G$ be a locally compact group, and let $p \in[1, \infty)$. Define $M^{p}(G)$ to be the completion of $M^{1}(G)$ in the norm given by

$\|\mu\|_{M^{p}(G)}=\sup \left\{\|\pi(\mu)\|: \pi: M^{1}(G) \rightarrow \mathcal{B}\left(L^{p}(\nu)\right)\right.$ is a contractive homomorphism $\}$ for $\mu \in M^{1}(G)$.

The algebra $M_{\lambda}^{p}(G)$ is the completion of $M^{1}(G)$ in the norm

$$
\|\mu\|_{M_{\lambda}^{p}(G)}=\left\|\lambda_{p}(\mu)\right\|_{\mathcal{B}\left(L^{p}(G)\right)}
$$

for $\mu \in M^{1}(G)$.

Remark 4.8. For $p \in[1, \infty)$, one can show that there are natural (isometric) inclusions

and

$$
F^{p}(G) \subseteq M^{p}(G) \subseteq M\left(F^{p}(G)\right)
$$

$$
F_{\lambda}^{p}(G) \subseteq M_{\lambda}^{p}(G) \subseteq M\left(F_{\lambda}^{p}(G)\right)
$$

Somewhat less easy is the existence of an inclusion $M_{\lambda}^{p}(G) \subseteq C V_{p}(G)$ for $p \in(1, \infty)$; see GT14. 
The following is a particular case of a result in GT14.

Proposition 4.9. Let $p \in[1, \infty)$, let $G$ be a locally compact group, and let $H \subseteq G$ be an amenable subgroup. Then the inclusion $H \hookrightarrow G$ induces canonical isometric unital homomorphisms

$$
M_{\lambda}^{p}(H) \rightarrow M_{\lambda}^{p}(G) \quad \text { and } \quad M^{p}(H) \rightarrow M^{p}(G) .
$$

The way in which the above proposition will be used is through the following lemma. We state it and prove it in greater generality than needed here for use elsewhere.

Lemma 4.10. Let $p \in[1, \infty)$ and let $G$ be a non-trivial locally compact group. Denote by $A^{p}(G)$ any of the following algebras: $F^{p}(G), M^{p}(G), F_{\lambda}^{p}(G), M_{\lambda}^{p}(G)$, $P M_{p}(G)$ or $C V_{p}(G)$ (the last two in the case $p>1$ ). If $A^{p}(G)$ is isometrically and non-degenerately representable on a Banach space $X$, then there exist a cyclic subgroup $H$ (finite or infinite) of $G$, and a unital isometric representation $\psi: F_{\lambda}^{p}(H) \rightarrow \mathcal{B}(X)$.

Proof. Choose a non-trivial element $g \in G$, and denote by $H$ the (not necessarily closed) cyclic subgroup of $G$ generated by $g$. Since either $F^{p}(G)$ or $F_{\lambda}^{p}(G)$ is isometrically a subalgebra of $A^{p}(G)$ (see Remark 4.8), we may assume, without loss of generality, that $A^{p}(G)$ is either $F_{\lambda}^{p}(G)$ or $F^{p}(G)$. Denote by $B^{p}(G)$ either $M_{\lambda}^{p}(G)$ (if $A^{p}(G)=F_{\lambda}^{p}(G)$ ) or $M^{p}(G)$ (if $A^{p}(G)=F^{p}(G)$ ). By Proposition 4.9. and since $H$ is amenable and discrete, the inclusion of $H \subseteq G$ induces a canonical isometric embedding $F_{\lambda}^{p}(H) \hookrightarrow B^{p}(G)$.

Let $X$ be a Banach space and let $\varphi: A^{p}(G) \rightarrow \mathcal{B}(X)$ be an isometric and nondegenerate representation. Note that $A^{p}(G)$ has a contractive approximate identity, since so does $L^{1}(G)$ and there is a contractive homomorphism $L^{1}(G) \rightarrow A^{p}(G)$ with dense image. By Theorem 4.5 $\varphi$ can be extended to an isometric unital representation $\psi: M\left(A^{p}(G)\right) \rightarrow \mathcal{B}(X)$. Since there are inclusions

$$
F_{\lambda}^{p}(H) \subseteq B^{p}(G) \subseteq M\left(A^{p}(G)\right),
$$

the restriction of $\psi$ to $F_{\lambda}^{p}(H)$ is the desired isometric unital representation of $F_{\lambda}^{p}(H)$ on $X$.

The next theorem will be needed in the proof of Theorem 4.12, Recall that the algebra $P M_{p}(G)$ of $p$-pseudomeasures is the closure of $F_{\lambda}^{p}(G)$ in $\mathcal{B}\left(L^{p}(G)\right)$ with respect to the weak* topology (also called the ultraweak topology) induced by the (canonical) identification of $\mathcal{B}\left(L^{p}(G)\right)$ with the dual of $L^{p}(G) \widehat{\otimes} L^{p^{\prime}}(G)$ given by the pairing determined on simple tensors by

$$
\langle a, \xi \otimes \eta\rangle_{\mathcal{B}\left(L^{p}(G)\right), L^{p}(G) \widehat{\otimes} L^{p^{\prime}}(G)}=\langle a \xi, \eta\rangle_{L^{p}(G), L^{p^{\prime}}(G)}
$$

for all $a \in \mathcal{B}\left(L^{p}(G)\right)$, all $\xi \in L^{p}(G)$ and all $\eta \in L^{p^{\prime}}(G)$.

We denote by $G^{\text {op }}$ the opposite group of $G$. With $\mu$ and $\nu$ as in subsection 1.1 the inversion map $\iota:(G, \mu) \rightarrow\left(G^{\text {op }}, \nu\right)$ is in fact a measure-preserving group isomorphism. In particular, the $p$-convolution algebras associated to $G$ are canonically isometrically isomorphic to those associated to $G^{\mathrm{op}}$.

Theorem 4.11. Let $G$ be a locally compact group, and let $p \in(1, \infty)$. Then there are canonical isometric isomorphisms

$$
F^{p}(G) \cong F^{p^{\prime}}(G), M^{p}(G) \cong M^{p^{\prime}}(G), F_{\lambda}^{p}(G) \cong F_{\lambda}^{p^{\prime}}(G),
$$




$$
M_{\lambda}^{p}(G) \cong M_{\lambda}^{p^{\prime}}(G), P M_{p}(G) \cong P M_{p^{\prime}}(G) \text { and } C V_{p}(G) \cong C V_{p^{\prime}}(G) .
$$

Proof. For $F^{p}(G)$, this is Proposition 2.18 in GT15b (here recalled as Proposition 2.4), while the proof for $M^{p}(G)$ is analogous, using $M^{1}(G)$ instead of $L^{1}(G)$.

For the reduced versions, we will show that there are canonical isometric isomorphisms $F^{p}(G) \cong F^{p^{\prime}}\left(G^{\mathrm{op}}\right), M_{\lambda}^{p}(G) \cong M_{\lambda}^{p^{\prime}}\left(G^{\mathrm{op}}\right), \quad P M_{p}(G) \cong P M_{p^{\prime}}\left(G^{\mathrm{op}}\right)$ and $C V_{p}(G) \cong C V_{p^{\prime}}\left(G^{\mathrm{op}}\right)$, since, by the remarks preceding this lemma, this implies the result.

From now on, and to minimize confusion, we will write $(G, \mu)$ and $\left(G^{\mathrm{op}}, \nu\right)$ instead of $G$ and $G^{\text {op }}$, to state explicitly which is the left Haar measure in each case.

Define an isometric anti-isomorphism $\phi: L^{1}(G, \mu) \rightarrow L^{1}\left(G^{\mathrm{op}}, \nu\right)$ by $\phi(f)(s)=$ $\Delta(s) f(s)$ for all $f \in L^{1}(G, \mu)$ and all $s \in G$. Observe that the assignment $\xi \mapsto$ $\xi \circ \iota$ defines an isometric isomorphism $L^{p^{\prime}}(G, \mu) \cong L^{p^{\prime}}\left(G^{\mathrm{op}}, \nu\right)$. We denote by $\psi: \mathcal{B}\left(L^{p}(G, \mu)\right) \rightarrow \mathcal{B}\left(L^{p^{\prime}}\left(G^{\text {op }}, \nu\right)\right)$ the map given by

$$
\psi(a)(\xi)(s)=\left[a^{\prime}(\xi \circ \iota)\right]\left(s^{-1}\right)
$$

for all $a \in \mathcal{B}\left(L^{p}(G, \mu)\right)$, for all $\xi \in L^{p^{\prime}}\left(G^{\mathrm{op}}, \mu\right)$ and for all $s \in G$. (In other words, $\psi(a)$ is the transpose of $a$, once $L^{p^{\prime}}(G, \mu)$ is identified with $L^{p^{\prime}}\left(G^{\text {op }}, \nu\right)$ via the assignment $\xi \mapsto \xi \circ \iota$.) It is easy to check that $\psi$ is an isometric anti-isomorphism.

We claim that the following diagram commutes:

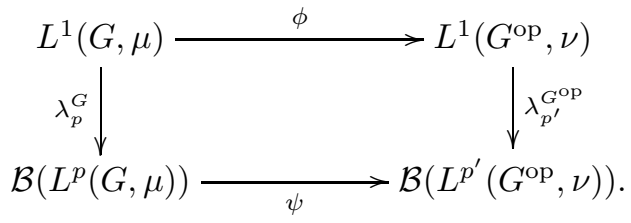

Let $f \in L^{1}(G)$, let $\xi \in L^{p^{\prime}}\left(G^{\text {op }}, \nu\right)$ and let $s \in G$ be given. Recall (see, for example, Proposition 3.5 in [GT15b]) that the transpose of $\lambda_{p}^{G}(f)$ is $\lambda_{p^{\prime}}^{G}(\phi(f) \circ \iota)$. (The function $\phi(f) \circ \iota \in L^{1}(G, \mu)$ is denoted by $f^{\sharp}$ in GT15b.) Using this at the second step, we get

$$
\begin{aligned}
\psi\left(\lambda_{p}^{G}(f)\right)(\xi)(s) & =\left[\lambda_{p}^{G}(f)^{\prime}(\xi \circ \iota)\right]\left(s^{-1}\right) \\
& =[(\phi(f) \circ \iota) *(\xi \circ \iota)]\left(s^{-1}\right) \\
& =\int_{G} \Delta\left(t^{-1}\right) f\left(t^{-1}\right) \xi(s t) d \mu(t) \\
& =\int_{G} f(t) \xi\left(s t^{-1}\right) d \mu(t) .
\end{aligned}
$$

On the other hand,

$$
\begin{aligned}
\lambda_{p^{\prime}}^{G^{\mathrm{op}}}(\phi(f))(\xi)(s) & =\left(\phi(f) *_{\mathrm{op}} \xi\right)(s) \\
& =\int_{G} \Delta(t) f(t) \xi\left(s t^{-1}\right) d \nu(t) \\
& =\int_{G} f(t) \xi\left(s t^{-1}\right) d \mu(t)
\end{aligned}
$$

which proves the claim. 
It follows that there is a canonical isometric isomorphism $F_{\lambda}^{p}(G) \cong F_{\lambda}^{p^{\prime}}\left(G^{\text {op }}\right)$, implemented by $\psi$. By taking double commutants, we deduce that $\psi$ also implements an isomorphism between $C V_{p}(G)$ and $C V_{p^{\prime}}(G)$. Extending the above diagram to $M^{1}(G, \mu)$ and $M^{1}\left(G^{\text {op }}, \nu\right)$, we conclude that there is also a canonical isometric isomorphism $M_{\lambda}^{p}(G) \cong M_{\lambda}^{p^{\prime}}\left(G^{\text {op }}\right)$.

To show the result for the algebras of pseudomeasures, we need to identify the ultraweak topologies on $\mathcal{B}\left(L^{p}(G, \mu)\right)$ and $\mathcal{B}\left(L^{p^{\prime}}\left(G^{\mathrm{op}}, \nu\right)\right)$. In order to do this, observe that the canonical isometric identification

$$
L^{p}(G, \mu) \widehat{\otimes} L^{p^{\prime}}(G, \mu) \cong L^{p^{\prime}}(G, \mu) \widehat{\otimes} L^{p}(G, \mu)
$$

induces the isometric isomorphism $\psi: \mathcal{B}\left(L^{p}(G, \mu)\right) \rightarrow \mathcal{B}\left(L^{p^{\prime}}(G, \mu)\right)$. Commutativity of the above diagram then implies that $\phi$ extends to an isometric isomorphism between the ultraweak closures of $L^{1}(G, \mu)$ and $L^{1}\left(G^{\text {op }}, \nu\right)$, that is, to an isometric isomorphism $P M_{p}(G) \cong P M_{p^{\prime}}\left(G^{\text {op }}\right)$.

The folowing is one of the main results of this paper. It determines precisely when one of the $p$-convolution algebras considered in the literature can be represented on an $L^{q}$-space, for some $q \in[1, \infty)$. It can be interpreted as stating that the $L^{p}$ and $L^{q}$-representation theories of a nontrivial group, are incomparable whenever they are not "obviously" equivalent. We point out that this represents a significant generalization of Theorem 2.2 in [NR09], where the authors only deal with the case $q=2$, and moreover assume that $G$ is amenable and has a closed infinite abelian subgroup.

The proof of Theorem 4.12 uses machinery from a number of other works. The reader is referred to the third page in the introduction for an overview of the proof.

Theorem 4.12. Let $G$ be a locally compact group, and let $p, q \in[1, \infty)$ with $q>$ 1. Then one (or all) of $F^{p}(G), M^{p}(G), F_{\lambda}^{p}(G), M_{\lambda}^{p}(G), P M_{p}(G)$ or $C V_{p}(G)$ is isometrically representable on an $L^{q}$-space if and only if one of the following holds:

(1) $G$ is the trivial group;

(2) we have $\left|\frac{1}{2}-\frac{1}{p}\right|=\left|\frac{1}{2}-\frac{1}{q}\right|$; or

(3) $p=2$ and $G$ is abelian.

Proof. We begin with the "if" implication. When $G$ is the trivial group, all the Banach algebras in the statement are $\mathbb{C}$, which is clearly representable on an $L^{q}$ space for any $q \in[1, \infty)$. On the other hand, the identity $\left|\frac{1}{2}-\frac{1}{p}\right|=\left|\frac{1}{2}-\frac{1}{q}\right|$ is equivalent to $p$ and $q$ being either equal or conjugate. The case $p=q$ is trivial. If $p$ and $q$ are conjugate, then Theorem 4.11 shows that all of the algebras in the statement are (canonically) representable on $L^{q}(G)$. Finally, if $p=2$ and $G$ is abelian, then all of the Banach algebras in the statement are commutative $C^{*}$ algebras, and hence have the form $C_{0}(X)$ for some locally compact Hausdorff space $X$. It is then an easy exercise to check that for any $q \in[1, \infty)$ and for any such space $X$, there exist an $L^{q}$-space and an isometric representation of $C_{0}(X)$ on it.

We turn to the "only if" implication. Since there are isometric inclusions

$F_{\lambda}^{p}(G) \subseteq M_{\lambda}^{p}(G) \subseteq C V_{p}(G), F_{\lambda}^{p}(G) \subseteq P M_{p}(G) \subseteq C V_{p}(G)$ and $F^{p}(G) \subseteq M^{p}(G)$

(see Remark 4.8), we may assume that either $F_{\lambda}^{p}(G)$ or $F^{p}(G)$ is representable on an $L^{q}$-space. By Proposition 2.4, and without loss of generality, we can assume that $p, q \in[1,2]$. Suppose that $p=2$ and $p \neq q$. Then $F_{\lambda}^{p}(G)=C_{\lambda}^{*}(G)$ and 
$F^{p}(G)=C^{*}(G)$ are $C^{*}$-algebras. By Theorem 4.4 these must be abelian $C^{*}$ algebras, whence $G$ itself must be abelian.

Since the arguments for $F_{\lambda}^{p}(G)$ and $F^{p}(G)$ are very similar, we will carry them out together until we have to distinguish the two cases. We therefore denote by $A^{p}(G)$ either $F_{\lambda}^{p}(G)$ or $F^{p}(G)$.

Now suppose that $p \neq 2$. Then $q \neq 2$ by Theorem 4.6. Let $E$ be an $L^{q}$-space and let $\varphi: A^{p}(G) \rightarrow \mathcal{B}(E)$ be an isometric representation. Note that $A^{p}(G)$ has a contractive approximate identity, since so does $L^{1}(G)$ and there is a contractive homomorphism $L^{1}(G) \rightarrow A^{p}(G)$ with dense image. By Theorem 2.2, there exists a contractive idempotent $e \in \mathcal{B}(E)$ such that $e(E)$ is the essential subspace $E_{0}$ of $\varphi$. Then $E_{0}$ is an $L^{q}$-space by Theorem 6 in [Tza69. Denote by $\varphi_{0}: A^{p}(G) \rightarrow \mathcal{B}\left(E_{0}\right)$ the restriction of $\varphi$. Then $\varphi_{0}$ is a non-degenerate isometric representation. By Lemma 4.10, there exist a cyclic subgroup $H$ of $G$, and an isometric unital representation $\psi: F_{\lambda}^{p}(H) \rightarrow \mathcal{B}\left(E_{0}\right)$. We claim that $\psi\left(F_{\lambda}^{p}(H)\right)$ is isometrically isomorphic to $F_{\lambda}^{q}(H)$. We divide the proof into two cases.

Case 1: $H \cong \mathbb{Z}$. Observe that $\psi\left(F_{\lambda}^{p}(\mathbb{Z})\right)$ and $F_{\lambda}^{q}(\mathbb{Z})$ are both Banach algebras generated by an invertible isometry of an $L^{q}$-space and its inverse. Since $q \neq 2$, by part $(2)$ of Corollary 5.21 in $\mathrm{GT} 15 \mathrm{a}, F_{\lambda}^{q}(\mathbb{Z})$ is the unique, up to (isometric) isomorphism, Banach algebra generated by an invertible isometry of an $L^{q_{-}}$ space and its inverse whose Gelfand transform is not an isomorphism (isometric or not). Since $p \neq 2$, the Gelfand transform of $F_{\lambda}^{p}(\mathbb{Z})$ is not an isomorphism, so the same is true for $\psi\left(F_{\lambda}^{p}(\mathbb{Z})\right)$. It thus follows that there is an isometric isomorphism $\psi\left(F_{\lambda}^{p}(H)\right) \cong F_{\lambda}^{q}(H)$, as desired. This proves the first case.

Case 2: $H \cong \mathbb{Z}_{r}$ for $r \geq 2$. By replacing $H$ with a subgroup, we may assume that $r$ is prime. Denote by $v \in F_{\lambda}^{p}\left(\mathbb{Z}_{r}\right)$ the canonical invertible isometry generating $F_{\lambda}^{p}\left(\mathbb{Z}_{r}\right)$, and set $w=\psi(v)$, which is an invertible isometry of $E_{0}$. Then

$$
\operatorname{sp}(w)=\operatorname{sp}(v)=\left\{\zeta \in S^{1}: \zeta^{r}=1\right\} .
$$

Thus, the only admissible spectral configurations $\sigma=\left(\sigma_{n}\right)_{n \in \mathbb{N}}$ for $w$ are

- $\sigma_{0}=\operatorname{sp}(w)$ and $\sigma_{n}=\emptyset$ for $n \geq 1$; or

- $\sigma_{0}=\operatorname{sp}(w)=\sigma_{r}$ and $\sigma_{n}=\emptyset$ for $n \geq 1$ with $n \neq r$.

In the first case, there is an isometric isomorphism between $F^{q}\left(w, w^{-1}\right)=$ $\psi\left(F_{\lambda}^{p}\left(\mathbb{Z}_{r}\right)\right)$ and $\left(\mathbb{C}^{r},\|\cdot\|_{\infty}\right)$. This would then contradict part (5) of Theorem 3.5 in GT15a, since $p \neq 2$. In the second case, by the definition of the norm $\|\cdot\|_{\sigma, q}$ on $F^{q}(\sigma) \cong F^{q}\left(w, w^{-1}\right)$, there is an isometric isomorphism $F_{\lambda}^{q}\left(\mathbb{Z}_{r}\right) \rightarrow \psi\left(F_{\lambda}^{p}\left(\mathbb{Z}_{r}\right)\right)$. This finishes the proof of the claim. For later use, we stress the fact that, in the case $H=\mathbb{Z}_{r}$, the isomorphism $\psi\left(F_{\lambda}^{p}(H)\right) \cong F_{\lambda}^{q}(H)$ is in fact canonical, in the sense that the spectral configurations of $v$ and $\psi(v)$ agree, and thus the isomorphism is induced by the identity on $\ell^{1}(H)$.

We deduce from the claim that there is an isometric isomorphism $F_{\lambda}^{p}(H) \cong$ $F_{\lambda}^{q}(H)$. For $H \cong \mathbb{Z}$, Theorem 3.6 implies that $p=q$. For $H \cong \mathbb{Z}_{r}$, we use the fact that the isomorphism can be chosen to be canonical to prove that $p$ must equal $q$.

So suppose that $p \neq q$ and $F_{\lambda}^{p}\left(\mathbb{Z}_{r}\right) \cong F_{\lambda}^{q}\left(\mathbb{Z}_{r}\right)$ canonically. Without loss of generality, we may assume that $1 \leq p<q<2$. We claim that the map $\gamma_{p, 2}: F_{\lambda}^{p}\left(\mathbb{Z}_{r}\right) \rightarrow$ $C_{\lambda}^{*}\left(\mathbb{Z}_{r}\right)$ from Theorem 2.5 is an isometric isomorphism. In view of Theorem 3.7 in 
GT15b, this is equivalent to showing that

$$
\left\|\lambda_{p}(f)\right\|_{\mathcal{B}\left(\ell^{p}\left(\mathbb{Z}_{r}\right)\right)}=\left\|\lambda_{2}(f)\right\|_{\mathcal{B}\left(\ell^{2}\left(\mathbb{Z}_{r}\right)\right)}
$$

for all $f \in \ell^{1}\left(\mathbb{Z}_{r}\right)$.

Let $f \in \ell^{1}\left(\mathbb{Z}_{r}\right)$. Then $\left\|\lambda_{2}(f)\right\|_{\mathcal{B}\left(\ell^{2}\left(\mathbb{Z}_{r}\right)\right)} \leq\left\|\lambda_{p}(f)\right\|_{\mathcal{B}\left(\ell^{p}\left(\mathbb{Z}_{r}\right)\right)}$ by Theorem 2.5. Let $\theta \in(0,1)$ satisfy $\frac{1}{q}=\frac{\theta}{p}+\frac{1-\theta}{2}$. By the Riesz-Thorin interpolation theorem, we have

$$
\left\|\lambda_{q}(f)\right\|_{\mathcal{B}\left(\ell^{q}\left(\mathbb{Z}_{r}\right)\right)} \leq\left\|\lambda_{2}(f)\right\|_{\mathcal{B}\left(\ell^{2}\left(\mathbb{Z}_{r}\right)\right)}^{\theta}\left\|\lambda_{p}(f)\right\|_{\mathcal{B}\left(\ell^{p}\left(\mathbb{Z}_{r}\right)\right)}^{1-\theta} .
$$

Since $\left\|\lambda_{p}(f)\right\|_{\mathcal{B}\left(\ell^{p}\left(\mathbb{Z}_{r}\right)\right)}=\left\|\lambda_{q}(f)\right\|_{\mathcal{B}\left(\ell^{q}\left(\mathbb{Z}_{r}\right)\right)}$, we conclude that

$$
\left\|\lambda_{p}(f)\right\|_{\mathcal{B}\left(\ell^{p}\left(\mathbb{Z}_{r}\right)\right)}^{\theta} \leq\left\|\lambda_{2}(f)\right\|_{\mathcal{B}\left(\ell^{2}\left(\mathbb{Z}_{r}\right)\right)}^{\theta}
$$

and hence $\left\|\lambda_{p}(f)\right\|_{\mathcal{B}\left(\ell^{p}\left(\mathbb{Z}_{r}\right)\right)} \leq\left\|\lambda_{2}(f)\right\|_{\mathcal{B}\left(\ell^{2}\left(\mathbb{Z}_{r}\right)\right)}$, as desired. This proves the claim.

Since $\gamma_{p, 2}$ is an isometric isomorphism, $F^{p}\left(\mathbb{Z}_{r}\right)$ is an operator algebra. The result now follows from Theorem 4.6

Corollary 4.13. Let $G$ be a locally compact group, and let $p, q \in[1, \infty)$. Then the following are equivalent:

(1) There is an isometric isomorphism $F^{p}(G) \cong F^{q}(G)$;

(2) There is an isometric isomorphism $F_{\lambda}^{p}(G) \cong F_{\lambda}^{q}(G)$;

(3) When $p>1$, there is an isometric isomorphism $P M_{p}(G) \cong P M_{q}(G)$;

(4) When $p>1$, there is an isometric isomorphism $C V_{p}(G) \cong C V_{q}(G)$;

(5) $\left|\frac{1}{p}-\frac{1}{2}\right|=\left|\frac{1}{q}-\frac{1}{2}\right|$.

In connection with GT16b], we mention here that for the algebras of pseudofunctions, the result above can be improved to moreover allow isomorphisms between algebras associated to different groups, as follows: for locally compact groups $G$ and $H$, and for $p, q \in[1, \infty)$ not both equal to 2 , there is an isometric isomorphism

$$
F_{\lambda}^{p}(G) \cong F_{\lambda}^{q}(H)
$$

if and only if $\left|\frac{1}{p}-\frac{1}{2}\right|=\left|\frac{1}{q}-\frac{1}{2}\right|$ and $G$ is isomorphic to $H$. (For discrete groups, the same result holds with $P M_{p}$ or $C V_{p}$ instead of $F_{\lambda}^{p}$.)

A variant of the techniques used to prove Theorem 4.12 can be used to rule out representability on certain $Q S L^{p}$-spaces. (Recall that a Banach space $E$ is a $Q S L^{p_{\text {- }}}$ space if it is isometrically isomorphic to a subspace of a quotient of an $L^{p}$-space. Group representations on $Q S L^{p}$-spaces are studied, for example, in [NR09.) We are not able to use spectral configurations as in Theorem 4.12 since we do not have a description of the Banach algebra generated by an invertible isometry of a $Q S L^{p}$-space. Instead, we will use the fact (see Theorem 3.7 in GT15b) that $F_{\lambda}^{p}\left(\mathbb{Z}_{n}\right)$ and $F_{\lambda}^{p}(\mathbb{Z})$ are universal with respect to representations on $Q S L^{p}$-spaces, together with the maps $\gamma_{p, q}$, to obtain the result.

The theorem below is only stated for $p, q \in[1,2]$, while the other exponents can be handled using duality.

Theorem 4.14. Let $G$ be a nontrivial locally compact group, and let $p, q \in[1,2]$.

(1) If $q \leq p$, then all of $F^{p}(G), M^{p}(G), F_{\lambda}^{p}(G), M_{\lambda}^{p}(G), P M_{p}(G)$ and $C V_{p}(G)$ can be isometrically represented on a $Q S L^{q}$-space.

(2) If $p<q$, then none of $F^{p}(G), M^{p}(G), F_{\lambda}^{p}(G), M_{\lambda}^{p}(G), P M_{p}(G)$ and $C V_{p}(G)$ can be isometrically represented on a $Q S L^{q}$-space. 
Proof. If $q \leq p$, then every $L^{p}$-space is isomorphic to a closed subspace of an $L^{q_{-}}$

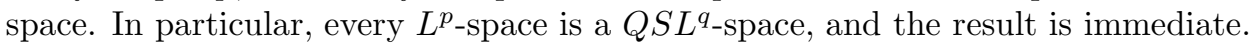

Suppose that $p<q$. As in the proof of Theorem 4.12, it is enough to show that $F^{p}(G)$ and $F_{\lambda}^{p}(G)$ are not representable on a $Q S L^{q}$-space. For convenience, we will denote by $A^{p}(G)$ one of these algebras, and by $B^{p}(G)$ either $M^{p}(G)$ or $M_{\lambda}^{p}(G)$, depending on which one $A^{p}(G)$ is denoting.

Let $E$ be a $Q S L^{q}$-space and let $\varphi: A^{p}(G) \rightarrow \mathcal{B}(E)$ be an isometric representation. Since a subspace of a $Q S L^{q}$-space is obviously a $Q S L^{q}$-space, we can assume that $\varphi$ is non-degenerate. By Lemma 4.10, there are a cyclic subgroup $H$ of $G$ and a unital, isometric representation $\psi: F_{\lambda}^{p}(H) \rightarrow \mathcal{B}(E)$. Unlike in the proof of Theorem 4.12, we cannot really conclude that $\psi\left(F_{\lambda}^{p}(H)\right)$ is isomorphic to $F_{\lambda}^{q}(H)$ directly. Instead, and using the notation from [GT15b], for $f \in \ell^{1}(H)$, we have (explanations below):

$$
\|f\|_{L^{p}}=\left\|\lambda_{p}(f)\right\|_{\mathcal{B}\left(L^{p}(H)\right)}=\|\psi(f)\|_{\mathcal{B}(E)} \leq\|f\|_{Q S L^{q}}=\|f\|_{L^{q}} \leq\|f\|_{L^{p}} .
$$

The first step is Theorem 3.7 in GT15b for $F^{p}(H)$ (the group $H$ is amenable); the second one is the fact that $\psi$ is isometric; the third one is the definition of the norm $\|\cdot\|_{Q S L^{q}}$; the fourth one is the fact (Theorem 3.7 in GT15b]) that $F_{\mathrm{QS}}^{p}(H)=F^{p}(H)$; and the last one is Theorem 2.5 .

It follows that the map $\gamma_{p, q}^{H}: F_{\lambda}^{p}(H) \rightarrow F_{\lambda}^{q}(H)$ is an isometric isomorphism. In particular, $F_{\lambda}^{p}(H)$ is representable on an $L^{q}$-space, which contradicts Theorem 4.12 , The contradiction implies that $A^{p}(G)$ cannot be isometrically represented on a $Q S L^{q}$-space, as desired.

We close this section with a question. Theorem 4.12 asserts that a group $C^{*}$ algebra can be represented on an $L^{p}$-space, for some $p \neq 2$, if and only if the group is commutative. More generally, Theorem 4.4 shows that a $C^{*}$-algebra can be represented on an $L^{p}$-space, for some $p \neq 2$, if and only if it is commutative. This may well be a particular case of a more general fact ruling out representability of certain $L^{p}$-operator algebras on $L^{q}$-spaces for two different, nonconjugate, Hölder exponents $p$ and $q$. On the other hand, the examples in [BLM95] show that there are operator algebras, which are not $C^{*}$-algebras, that can be represented on $L^{p}$-spaces for $p \neq 2$. Therefore, we suggest:

Question 4.15. Let $A$ be a unital Banach algebra, and suppose that the set

$$
\left\{v \in A: v \text { is invertible and }\|v\|=\left\|v^{-1}\right\|=1\right\}
$$

has dense linear span in $A$. (This guarantees that $A$ is a $C^{*}$-algebra if it is an operator algebra.) Let $p, q \in[1,2]$ with $p \neq q$. Suppose that $A$ can be isometrically represented on an $L^{p}$-space and on an $L^{q}$-space. Is $A$ commutative? Does it follow that $A \cong C(X)$ for some compact Hausdorff space $X$ ?

\section{An Application to CROSSED PRODUCTS}

Let $X$ be a locally compact Hausdorff space, let $G$ be a locally compact group, and let $\alpha: G \rightarrow \operatorname{Homeo}(X)$ be a topological action. For $p \in[1, \infty)$, N. Christopher Phillips defined in Phi13] the full and the reduced $L^{p}$-operator crossed products $F^{p}(G, X, \alpha)$ and $F_{\lambda}^{p}(G, X, \alpha)$ of the topological dynamical system $(G, X, \alpha)$, generalizing the well established constructions in $C^{*}$-algebras, which are the case $p=2$.

In Question 8.2 of Phi13, Phillips asked whether, for a topological dynamical system $(G, X, \alpha)$ and for distinct $p, q \in[1, \infty)$, there are any non-zero continuous 
homomorphisms

$$
F^{p}(G, X, \alpha) \rightarrow F^{q}(G, X, \alpha) \quad \text { or } \quad F_{\lambda}^{p}(G, X, \alpha) \rightarrow F_{\lambda}^{q}(G, X, \alpha) .
$$

While Theorem 2.5 shows that there may in general exist such homomorphisms (even contractive ones with dense range), in Corollary 5.11 we show that there is an isometric isomorphism $F^{p}(G, X, \alpha) \rightarrow F^{q}(G, X, \alpha)$ or $F_{\lambda}^{p}(G, X, \alpha) \rightarrow F_{\lambda}^{q}\left(G^{\mathrm{op}}, X, \alpha\right)$ if and only if $\left|\frac{1}{p}-\frac{1}{2}\right|=\left|\frac{1}{q}-\frac{1}{2}\right|$.

For the convenience of the reader, and since our notation is somewhat different, we recall below the definitions of the crossed products (for $L^{p}$-operator algebras other than $\left.C_{0}(X)\right)$. Recall that for $p \in[1, \infty)$, we say that a Banach algebra $A$ is an $L^{p}$-operator algebra if it can be isometrically represented on an $L^{p}$-space. The group of isometric automorphisms of $A$ is denoted $\operatorname{Aut}(A)$, and is always endowed with the strong topology.

The object we define next is the analog of the group algebra $L^{1}(G)$. All integrals are taken with respect to a fixed left Haar measure.

Definition 5.1. Fix $p \in[1, \infty)$. Let $\alpha: G \rightarrow \operatorname{Aut}(A)$ be an action of a locally compact group $G$ on a Banach algebra $A$. Denote by $L^{1}(G, A, \alpha)$ the Banach algebra completion of the space of continuous compactly supported functions $G \rightarrow A$ with respect to the $L^{1}$-norm, with product given by twisted convolution, that is,

$$
(a * b)(g)=\int_{G} a(h) \alpha_{h}\left(b\left(h^{-1} g\right)\right) d h
$$

for $a, b \in C_{c}(G, A, \alpha) \subseteq L^{1}(G, A, \alpha)$ and $g \in G$.

Next, we define (regular) covariant representations and the associated (reduced) crossed product.

Definition 5.2. Adopt the notation from the previous definition. A (contractive) covariant representation of $(G, A, \alpha)$ on an $L^{p}$-space $E$ is a pair $(\pi, v)$ consisting of a nondegenerate contractive homomorphism $\pi: A \rightarrow \mathcal{B}(E)$ and an isometric group representation $v: G \rightarrow \mathcal{B}(E)$, satisfying the covariance condition

$$
v_{g} \pi(a) v_{g^{-1}}=\pi\left(\alpha_{g}(a)\right)
$$

for all $g \in G$ and for all $a \in A$. Given such a covariant representation, its integrated form is the nondegenerate contractive homomorphism $\pi \rtimes v: L^{1}(G, A, \alpha) \rightarrow \mathcal{B}(E)$ given by

$$
(\pi \rtimes v)(a)(\xi)=\int_{G} \pi(a(g)) v_{g}(\xi) d g
$$

for all $a \in L^{1}(G, A, \alpha)$ and for all $\xi \in E$.

Given a contractive nondegenerate representation $\pi_{0}: A \rightarrow \mathcal{B}\left(E_{0}\right)$ on an $L^{p_{-}}$ space $E_{0}$, its associated regular covariant representation is the pair $\left(\pi, \lambda_{p}^{E_{0}}\right)$ on $L^{p}(G) \otimes_{p} E_{0} \cong L^{p}\left(G, E_{0}\right)$ given by

$$
\pi(a)(\xi)(g)=\pi_{0}\left(\alpha_{g^{-1}}(a)\right)(\xi(g)) \text { and }\left(\lambda_{p}^{E_{0}}\right)_{g}(\xi)(h)=\xi\left(g^{-1} h\right)
$$

for all $a \in A$, for all $\xi \in L^{p}\left(G, E_{0}\right)$, and for all $g, h \in G$.

Denote by $\operatorname{Rep}_{p}(G, A, \alpha)$ the class of all contractive covariant representations of $(G, A, \alpha)$ on $L^{p}$-spaces, and by $\operatorname{RegRep}_{p}(G, A, \alpha)$ the subclass of $\operatorname{Rep}_{p}(G, A, \alpha)$ consisting of the regular covariant representations. The full crossed product $F^{p}(G, A, \alpha)$ 
and the reduced crossed product $F_{\lambda}^{p}(G, A, \alpha)$ are defined as the completions of $L^{1}(G, A, \alpha)$ in the following norms:

$$
\|a\|_{F^{p}(G, A, \alpha)}=\sup \left\{\|(\pi \rtimes v)(a)\|:(\pi, v) \in \operatorname{Rep}_{p}(G, A, \alpha)\right\}
$$

and

$$
\|a\|_{F_{\lambda}^{p}(G, A, \alpha)}=\sup \left\{\left\|\left(\pi \rtimes \lambda_{p}^{E_{0}}\right)(a)\right\|:\left(\pi, \lambda_{p}^{E_{0}}\right) \in \operatorname{RegRep}_{p}(G, A, \alpha)\right\},
$$

for all $a \in L^{1}(G, A, \alpha)$.

Remark 5.3. In the context of the above definition, if $A$ is an $L^{p}$-operator algebra, then so will be $F^{p}(G, A, \alpha)$ and $F_{\lambda}^{p}(G, A, \alpha)$.

$L^{p}$-operator crossed products generalize group $L^{p}$-operator algebras, since for the one point space $X=\{*\}$ we have $F^{p}(G, *, \mathrm{id})=F^{p}(G)$ and $F_{\lambda}^{p}(G, *$, id $)=F_{\lambda}^{p}(G)$.

Remark 5.4. Most results in Phi13 assume that the algebra $A$ is separable and that the group is second countable, and conclude that a number of Banach algebras can be represented on $\sigma$-finite $L^{p}$-spaces; see also Remark 1.18 in Phi13. (The purpose of using $\sigma$-finite measure spaces is to apply Lamperti's theorem [Lam58.) However, the theory can be developed without these countability assumptions, and in fact the arguments in Phi13 go through in general, except that one gets an $L^{p_{-}}$ operator algebra that is not necessarily representable on a $\sigma$-finite $L^{p}$-space. This, in particular, applies to Remark 4.6 and Proposition 4.8 in [Phi13.

We note that while Definition 5.2 assumes covariant representations to be nondegenerate, this assumption is unnecessary whenever $A$ has a (left or right) contractive approximate identity, as can be shown with essentially the same argument as in Proposition 2.3 In fact, our first preparatory result guarantees the existence of an approximate identity for full and reduced crossed products whenever the underlying algebra has one.

Theorem 5.5. Let $p \in[1, \infty)$, let $A$ be an $L^{p}$-operator algebra, let $G$ be a locally compact group, and let $\alpha: G \rightarrow \operatorname{Aut}(A)$ be an action. Consider the following statements.

(1) A has a left (right, two-sided) contractive approximate identity;

(2) $F^{p}(G, A, \alpha)$ has a left (right, two-sided) contractive approximate identity;

(3) $F_{\lambda}^{p}(G, A, \alpha)$ has a left (right, two-sided) contractive approximate identity.

Then (1) implies (2), and (2) implies (3).

Finally, if $G$ is discrete, then also (3) implies (1), so they are all equivalent in this case.

Proof. We prove the result for left contractive approximate identities; the proof for right contractive approximate identities is analogous.

(1) implies (2). Let $\left(a_{\mu}\right)_{\mu \in \Lambda}$ be a contractive approximate identity for $A$. Set

$$
\mathcal{U}=\{U \subseteq G \text { open, such that } e \in U \text { and } \bar{U} \text { is compact }\} .
$$

Given $U \in \mathcal{U}$, let $f_{U}: G \rightarrow[0, \infty)$ be a continuous positive function with support contained in $U$ satisfying $\int_{G} f_{U}(g) d g=1$. Define a partial order on $\mathcal{U}$ by setting $U \leq V$ if $V \subseteq U$, and give $\mathcal{U} \times \Lambda$ the partial order given by $(U, \mu) \leq(V, \nu)$ if and only if $U \leq V$ and $\mu \leq \nu$. Recall that there exists a canonical contractive homomorphism $\iota: L^{1}(G, A, \alpha) \rightarrow F^{p}(G, A, \alpha)$ with dense range. Write $\|\cdot\|_{1}$ for 
the norm on both $L^{1}(G)$ and $L^{1}(G, A, \alpha)$, and $\|\cdot\|$ for the norm on both $A$ and $F^{p}(G, A, \alpha)$.

For $(U, \mu) \in \mathcal{U} \times \Lambda$, set $x_{(U, \mu)}=f_{U} a_{\mu}$ (pointwise product), which is an element in $C_{c}(G, A, \alpha) \subseteq L^{1}(G, A, \alpha)$. It is clear that

$$
\left\|\iota\left(x_{(U, \mu)}\right)\right\| \leq\left\|f_{U} a_{\mu}\right\|_{1}=\left\|f_{U}\right\|_{1}\left\|a_{\mu}\right\| \leq 1
$$

for all $(U, \mu) \in \mathcal{U} \times \Lambda$. Thus, in order to show that $\left(\iota\left(x_{(U, \mu)}\right)\right)_{(U, \mu) \in \mathcal{U} \times \Lambda}$ is a left contractive approximate identity for $F^{p}(G, A, \alpha)$, it is enough to show that $\left(x_{(U, \mu)}\right)_{(U, \mu) \in \mathcal{U} \times \Lambda}$ is a left contractive approximate identity for $L^{1}(G, A, \alpha)$. For this, it is enough to consider functions in $L^{1}(G) \cdot A \subseteq L^{1}(G, A, \alpha)$, since these span a dense subalgebra.

Let $f \in L^{1}(G)$ and $a \in A$ be given. We claim that $\left\|x_{(U, \mu)} *(f a)-f a\right\| \rightarrow 0$ as $(U, \mu) \rightarrow \infty$. If either $f$ or $a$ is zero, then so is their product and there is nothing to show. Without loss of generality, we may assume that $\|f\|_{1}=\|a\|=1$. Let $\varepsilon>0$. We make the following choices:

(1) Using continuity of $\alpha$, choose an open set $U_{1} \subseteq G$ containing the unit of $G$ such that $\left\|\alpha_{g}(a)-a\right\|<\varepsilon / 3$ for all $g \in U_{1}$. Since $G$ is locally compact, we may assume that $U_{1}$ has compact closure, so that $U_{1} \in \mathcal{U}$.

(2) Using continuity of the left regular representation on $L^{1}(G)$, choose an open set $U_{2} \subseteq G$ containing the unit of $G$ such that $\int_{G}\left|f\left(g^{-1} h\right)-f(h)\right| d g<\varepsilon / 3$ for all $g \in U_{2}$. Again, we may assume that $U_{2} \in \mathcal{U}$.

(3) Choose $\mu_{0} \in \Lambda$ such that $\left\|a_{\mu} a-a\right\|<\varepsilon / 3$ for all $\mu \geq \mu_{0}$.

Set $U_{0}=U_{1} \cap U_{2} \in \mathcal{U}$. Given $(U, \mu) \geq\left(U_{0}, \mu_{0}\right)$, we have

$$
\begin{aligned}
\left\|x_{(U, \mu)} *(f a)-f a\right\|= & \int_{G}\left\|\int_{G} x_{(U, \mu)}(g) \alpha_{g}\left((f a)\left(g^{-1} h\right)\right) d g-f(h) a\right\| d h \\
= & \int_{G}\left\|\int_{G} f_{U}(g) a_{\mu} f\left(g^{-1} h\right) \alpha_{g}(a) d g-f(h) a\right\| d h \\
= & \int_{G}\left\|\int_{G} f_{U}(g)\left(f\left(g^{-1} h\right) a_{\mu} \alpha_{g}(a)-f(h) a\right) d g\right\| d h \\
\leq & \int_{G} \int_{G} f_{U}(g)\left\|f\left(g^{-1} h\right) a_{\mu} \alpha_{g}(a)-f(h) a\right\| d g d h \\
\leq & \int_{G} \int_{G} f_{U}(g)\left\|f\left(g^{-1} h\right) a_{\mu} \alpha_{g}(a)-f(h) a_{\mu} \alpha_{g}(a)\right\| d g d h \\
& +\int_{G} \int_{G} f_{U}(g)\left\|f(h) a_{\mu} \alpha_{g}(a)-f(h) a_{\mu} a\right\| d g d h \\
& +\int_{G} \int_{G} f_{U}(g)\left\|f(h) a_{\mu} a-f(h) a\right\| d g d h \\
\leq & \left\|a_{\mu}\right\|\left\|\alpha_{g}(a)\right\| \int_{G} \int_{U} f_{U}(g)\left|f\left(g^{-1} h\right)-f(h)\right| d h d g \\
& +\left\|a_{\mu}\right\|\|f\|_{1} \int_{U} f_{U}(g)\left\|\alpha_{g}(a)-a\right\| d g+\left\|f_{U}\right\|_{1}\|f\|_{1}\left\|a_{\mu} a-a\right\| \\
\leq & \varepsilon / 3+\varepsilon / 3+\varepsilon / 3=\varepsilon
\end{aligned}
$$

We conclude that $F^{p}(G, A, \alpha)$ has a left contractive approximate identity. 
(2) implies (3). This is immediate since there is a contractive homomorphism $\kappa_{p}: F^{p}(G, A, \alpha) \rightarrow F_{\lambda}^{p}(G, A, \alpha)$ with dense range; see Lemma 3.13 in Phi13.

Assume now that $G$ is discrete, and let us prove that (3) implies (1). Denote by $E: F_{\lambda}^{p}(G, A, \alpha) \rightarrow A$ the faithful conditional expectation constructed in Proposition 4.8 of Phi13 (observe that separability is not necessary; see comments above). Let $\left(x_{\mu}\right)_{\mu \in \Lambda}$ be a contractive left approximate identity for $F_{\lambda}^{p}(G, A, \alpha)$, and set $y_{\mu}=E\left(x_{\mu}\right) \in A$ for $\mu \in \Lambda$. Using Remark 4.6 in Phi13 (again, separability is not needed there), we identify $A$ with a subalgebra of $F_{\lambda}^{p}(G, A, \alpha)$. For $a \in A$, we have

$$
\lim _{\mu \in \Lambda}\left\|y_{\mu} a-a\right\|=\lim _{\mu \in \Lambda}\left\|E\left(x_{\mu}\right) a-a\right\|=\lim _{\mu \in \Lambda}\left\|E\left(x_{\mu} a-a\right)\right\| \leq \lim _{\mu \in \Lambda}\left\|x_{\mu} a-a\right\|=0 .
$$

It follows that $\left(y_{\mu}\right)_{\mu \in \Lambda}$ is an left approximate identity for $A$, and it is clear that $\left\|y_{\mu}\right\| \leq 1$ for all $\mu \in \Lambda$.

Even when $G$ is discrete, the crossed products $F^{p}(G, A, \alpha)$ and $F_{\lambda}^{p}(G, A, \alpha)$ from the proposition above may not contain $F^{p}(G)$ and $F_{\lambda}^{p}(G)$ canonically; this happens only when $A$ is unital. In general, however, they are canonically subalgebras of the multiplier algebras of the crossed products, as we show below. Recall our convention that the product in multiplier algebras is written with a dot.

Theorem 5.6. Let $p \in[1, \infty)$, let $A$ be an $L^{p}$-operator algebra with a left contractive approximate identity, let $G$ be a locally compact group, and let $\alpha: G \rightarrow$ $\operatorname{Aut}(A)$ be an action by isometric isomorphisms. For $g \in G$, define a linear map $L_{g}: L^{1}(G, A, \alpha) \rightarrow L^{1}(G, A, \alpha)$ by

$$
L_{g}(a)(h)=\alpha_{g}\left(a\left(g^{-1} h\right)\right)
$$

for all $a \in L^{1}(G, A, \alpha)$ and all $h \in G$. Then the assignment $g \mapsto L_{g}$ induces natural contractive homomorphisms

$$
\iota_{L}^{G}: F^{p}(G) \rightarrow M_{L}\left(F^{p}(G, A, \alpha)\right) \text { and } \iota_{L, \lambda}^{G}: F_{\lambda}^{p}(G) \rightarrow M_{L}\left(F_{\lambda}^{p}(G, A, \alpha)\right) .
$$

When $A$ has a right contractive approximate identity, for $g \in G$, the linear maps $R_{g}: L^{1}(G, A, \alpha) \rightarrow L^{1}(G, A, \alpha)$, given by $R_{g}(a)(h)=a(h g)$ for all $a \in L^{1}(G, A, \alpha)$ and all $h \in H$, define natural contractive homomoprhisms

$$
\iota_{R}^{G}: F^{p}(G) \rightarrow M_{R}\left(F^{p}(G, A, \alpha)\right) \text { and } \iota_{R, \lambda}^{G}: F_{\lambda}^{p}(G) \rightarrow M_{R}\left(F_{\lambda}^{p}(G, A, \alpha)\right) .
$$

Finally, when A has a two-sided contractive approximate identity, then the above maps define natural contractive homomorphisms

$$
\iota^{G}: F^{p}(G) \rightarrow M\left(F^{p}(G, A, \alpha)\right) \text { and } \iota_{\lambda}^{G}: F_{\lambda}^{p}(G) \rightarrow M\left(F_{\lambda}^{p}(G, A, \alpha)\right) .
$$

Moreover, the maps $\iota_{L, \lambda}^{G}, \iota_{R, \lambda}^{G}$ and $\iota_{\lambda}^{G}$ are isometric.

Proof. We prove the theorem only for left approximate identities and left multiplier algebras, but analogous proofs apply to the right and two-sided versions. 
We claim that $L_{g}$ is a left multiplier on $L^{1}(G, A, \alpha)$, that is, $L_{g}(a * b)=L_{g}(a) * b$ for all $a, b \in L^{1}(G, A, \alpha)$. Given $a, b \in L^{1}(G, A, \alpha)$ and $h \in G$, we have

$$
\begin{aligned}
L_{g}(a * b)(h) & =\alpha_{g}\left(\int_{G} a(k) \alpha_{k}\left(b\left(k^{-1} g^{-1} h\right)\right) d k\right) \\
& =\int_{G} \alpha_{g}(a(k)) \alpha_{g k}\left(b\left((g k)^{-1} h\right)\right) d k \\
& =\int_{G} \alpha_{g}\left(a\left(g^{-1} t\right)\right) \alpha_{t}\left(b\left(t^{-1} h\right)\right) d t \\
& =\left(L_{g}(a) * b\right)(h),
\end{aligned}
$$

as desired. It is clear that $g \mapsto L_{g}$ defines a strongly continuous group homomorphism $L: G \rightarrow M_{L}\left(L^{1}(G, A, \alpha)\right)$.

Claim 1: The homomorphism $L$ extends to strongly continuous representations, $\iota^{G}$ and $\iota_{\lambda}^{G}$, of $G$ on $F^{p}(G, A, \alpha)$ and $F_{\lambda}^{p}(G, A, \alpha)$ by isometric left multipliers.

Let $g \in G$ be given. To show that $L_{g}$ extends to isometric automorphisms $\iota_{g}^{G}$ and $\left(\iota_{\lambda}^{G}\right)_{g}$ of $F^{p}(G, A, \alpha)$ and $F_{\lambda}^{p}(G, A, \alpha)$, it is enough to show that for every covariant representation $(\pi, v)$ of $(G, A, \alpha)$ on an $L^{p}$-space, one has

$$
\|(\pi \rtimes v)(a)\|=\left\|(\pi \rtimes v)\left(L_{g}(a)\right)\right\|
$$

for all $a \in L^{1}(G, A, \alpha)$. To prove this, let $(\pi, v)$ be a covariant representation on an $L^{p}$-space $E$, and let $a \in L^{1}(G, A, \alpha)$. For $\xi \in E$, we use the covariance identity $\pi\left(\alpha_{g}(x)\right)=v_{g} \pi(x) v_{g^{-1}}$ at the third step, to get

$$
\begin{aligned}
(\pi \rtimes v)\left(L_{g}(a)\right)(\xi) & =\int_{G} \pi\left(L_{g}(a)(h)\right) v_{h}(\xi) d h \\
& =\int_{G} \pi\left(\alpha_{g}\left(a\left(g^{-1} h\right)\right)\right) v_{h}(\xi) d h \\
& =\int_{G} v_{g} \pi\left(a\left(g^{-1} h\right)\right) v_{g^{-1} h}(\xi) d h \\
& =v_{g}(\pi \rtimes v)(a)(\xi) .
\end{aligned}
$$

Since $v_{g}$ is an isometry, it follows that $\|(\pi \rtimes v)(a)\|=\left\|(\pi \rtimes v)\left(L_{g}(a)\right)\right\|$, as desired.

The fact that $\varphi_{g}$ and $\left(\iota_{L, \lambda}^{G}\right)_{g}$ are left multipliers follows immediately from the fact that $L_{g}$ is a left multiplier, using an $\varepsilon / 3$ argument. Finally, it is routine to check that the assignments $g \mapsto\left(\iota_{L}^{G}\right)_{g}$ and $g \mapsto\left(\iota_{L, \lambda}^{G}\right)_{g}$ are strongly continuous actions of $G$. The claim is proved.

Since $L^{1}(G)$ is universal with respect to strongly continuous isometric actions of $G$, there are contractive homomorphisms $\iota_{L}^{G}: L^{1}(G) \rightarrow M_{L}\left(F^{p}(G, A, \alpha)\right)$ and $\iota_{L, \lambda}^{G}: L^{1}(G) \rightarrow M_{L}\left(F_{\lambda}^{p}(G, A, \alpha)\right)$, which admit explicit descriptions as follows. For $f \in L^{1}(G)$ and $a \in L^{1}(G, A, \alpha)$, we have

$$
\left(\iota_{L}^{G}(f) \cdot a\right)(g)=\int_{G} f(h) L_{h}(a)(g) d h=\int_{G} f(h) \alpha_{h}\left(a\left(h^{-1} g\right)\right) d h,
$$

for all $g \in G$. On the other hand, let $\kappa: F^{p}(G, A, \alpha) \rightarrow F_{\lambda}^{p}(G, A, \alpha)$ denote the canonical contractive homomorphism with dense range. Since $\kappa$ is the identity on $L^{1}(G, A, \alpha)$, by Theorem 5.5 it maps a contractive left approximate identity of $F^{p}(G, A, \alpha)$ to a contractive left approximate identity of $F_{\lambda}^{p}(G, A, \alpha)$. Thus there exists a unique unital extension $\widetilde{\kappa}: M_{L}\left(F^{p}(G, A, \alpha)\right) \rightarrow M_{L}\left(F_{\lambda}^{p}(G, A, \alpha)\right)$, and we have $\iota_{L, \lambda}^{G}=\widetilde{\kappa} \circ \iota_{L}^{G}$. 
It remains to show that $\iota_{L, \lambda}^{G}$ is isometric when $L^{1}(G)$ is endowed with the norm of $F_{\lambda}^{p}(G)$. We begin with some general observations. Since $F^{p}(G, A, \alpha)$ and $F_{\lambda}^{p}(G, A, \alpha)$ have left contractive approximate identities by Theorem 5.5, it follows from Theorem 4.5 that any contractive, nondegenerate representation of any these algebras extends to a contractive, unital representation of its left multiplier algebra. Let $(\pi, v)$ be a covariant representation of $(G, A, \alpha)$ on an $L^{p}$-space $E$. By applying Theorem 2.2, we can assume that $\pi$ (and hence $\pi \rtimes v$ ) is nondegenerate. We write $\widetilde{\pi \rtimes v}: M_{L}\left(F^{p}(G, A, \alpha)\right) \rightarrow \mathcal{B}(E)$ for the extension of $\pi \rtimes v$ to the left multiplier algebra, and similarly with $\widetilde{\pi \rtimes v}: M_{L}\left(F_{\lambda}^{p}(G, A, \alpha)\right) \rightarrow \mathcal{B}(E)$ if $(\pi, v)$ is a regular covariant representation. By a slight abuse of notation, we denote also by $v: L^{1}(G) \rightarrow \mathcal{B}(E)$ the integrated form of $v$, which is given by $v(f) \xi=\int_{G} f(g) v_{g}(\xi) d g$ for $f \in L^{1}(G)$ and $\xi \in E$.

Claim 2: We have $\widetilde{\pi \rtimes v}\left(\iota_{L}^{G}(f)\right)=v(f)$ for all $f \in L^{1}(G)$. Let $f \in L^{1}(G)$, let $a \in L^{1}(G, A, \alpha)$, and let $\xi \in E$. Then

$$
\begin{aligned}
& (\widetilde{\pi \rtimes v})\left(\iota_{L}^{G}(f)\right)[(\pi \rtimes v)(a) \xi]=(\pi \rtimes v)\left[\iota_{L}^{G}(f) \cdot a\right](\xi) \\
& =\int_{G} \pi\left[\left(\iota_{L}^{G}(f) \cdot a\right)(g)\right] v_{g}(\xi) d g \\
& =\int_{G} \pi\left[\int_{G} f(h) \alpha_{h}\left(a\left(h^{-1} g\right)\right) d h\right] v_{g}(\xi) d g \\
& =\int_{G} f(h) \int_{G} \pi\left[\alpha_{h}\left(a\left(h^{-1} g\right)\right)\right] v_{g}(\xi) d g d h \\
& =\int_{G} f(h) \int_{G} \pi\left[\alpha_{h}(a(k))\right] v_{h k}(\xi) d k d h \\
& =\int_{G} f(h) \int_{G} v_{h} \pi[a(k)] v_{k}(\xi) d k d h \\
& =\int_{G} f(h) v_{h}((\pi \rtimes v)(a) \xi) d h \\
& =v(f)((\pi \rtimes v)(a) \xi),
\end{aligned}
$$

and the claim is proved.

Now suppose that $(\pi, v)$ is a regular covariant representation, so that there exists an $L^{p}$-space $E_{0}$ such that $v=\lambda_{p}^{E_{0}}$; see Definition 5.2. Let $f \in L^{1}(G)$, and observe that

$$
\left\|\lambda_{p}^{E_{0}}(f)\right\|=\left\|\lambda_{p}(f) \otimes \operatorname{id}_{E_{0}}\right\|=\left\|\lambda_{p}(f)\right\|
$$

Using Claim 2 at the second step, and the above identity at the third step, we get

$$
\begin{aligned}
\left\|\iota_{L, \lambda}^{G}(f)\right\|_{M\left(F_{\lambda}^{p}(G, A, \alpha)\right)} & =\sup \left\{\left\|\left(\pi \rtimes \lambda_{p}^{E_{0}}\right)\left(\iota_{L}^{G}(f)\right)\right\|:\left(\pi, \lambda_{p}^{E_{0}}\right) \in \operatorname{RegRep}_{p}(G, A, \alpha)\right\} \\
& =\sup \left\{\left\|\lambda_{p}^{E_{0}}(f)\right\|:\left(\pi, \lambda_{p}^{E_{0}}\right) \in \operatorname{RegRep}_{p}(G, A, \alpha)\right\} \\
& =\left\|\lambda_{p}(f)\right\| \\
& =\|f\|_{F_{\lambda}^{p}(G)} .
\end{aligned}
$$

We conclude that $\iota_{L, \lambda}^{G}: F_{\lambda}^{p}(G) \rightarrow M_{L}\left(F_{\lambda}^{p}(G, A, \alpha)\right)$ is isometric. This finishes the proof.

When $p=1$, and regardless of whether $G$ is amenable or not, there is a canonical identification $F^{1}(G)=F_{\lambda}^{1}(G)$ (see Proposition 2.11 in GT15b]), and hence the 
maps $\iota_{L}^{G}, \iota_{R}^{G}$ and $\iota^{G}$ from Theorem 5.6 are isometric (because they agree with the ones defined on $F_{\lambda}^{1}(G)$ ). However, when $p>1$, it is not in general true that the maps defined on $F^{p}(G)$ are isometric, or even injective with closed range, as we explain in the following example.

Example 5.7. Let $G$ be a discrete group, and let Lt denote the action of $G$ on $c_{0}(G)$ by left translation. By Theorem 4.3 in GT15b, the canonical map $F^{p}(G, G, \mathrm{Lt}) \rightarrow F_{\lambda}^{p}(G, G, \mathrm{Lt})$ is an isometric isomorphism, regardless of $G$. Denote by $\kappa_{p}: F^{p}(G) \rightarrow F_{\lambda}^{p}(G)$ the canonical contractive map with dense range, and recall (Theorem 2.7) that $\kappa_{p}$ is a (not necessarily isometric) isomorphism if and only if $G$ is amenable. Naturality of the maps involved implies that the following diagram of unital homomorphisms commutes:

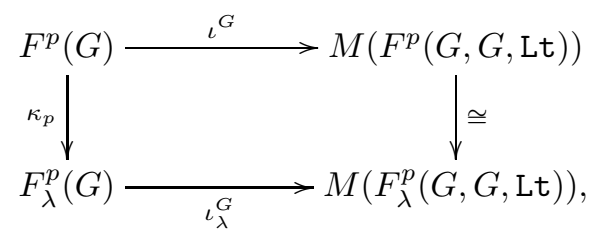

where the vertical map on the right is the canonical one. Now, if $\iota^{G}$ were injective and had closed range, that is, if it were an isomorphism onto its range, then it would follow that $\kappa_{p}$ is an isomorphism, and hence that $G$ is amenable.

Since an identical reasoning applies to left or right multiplier algebras, we conclude that for any non-amenable group $G$, and for the action Lt, the maps $\iota_{L}^{G}, \iota_{R}^{G}$ and $\iota^{G}$ are not isomorphisms onto their ranges.

Let $\alpha: G \rightarrow \operatorname{Aut}(A)$ be an action of a locally compact group $G$ on a Banach algebra $A$. We denote by $A^{\text {op }}$ the opposite Banach algebra, and write $\alpha^{\mathrm{op}}: G \rightarrow$ $\operatorname{Aut}\left(A^{\mathrm{op}}\right)$ for the action given by $\alpha_{g}^{\mathrm{op}}=\alpha_{g}$ for all $g \in G$. When $A$ is abelian, then clearly $A=A^{\text {op }}$ and $\alpha=\alpha^{\text {op }}$.

We note that in $L^{1}\left(G, A^{\text {op }}, \alpha^{\text {op }}\right)$, convolution is performed using opposite product on $A$, which we denote by op to minimize confusion. Recall that $\Delta: G \rightarrow \mathbb{R}_{+}$ denotes the modular function (see subsection 1.1).

Proposition 5.8. Let $p \in(1, \infty)$, let $A$ be an $L^{p}$-operator algebra, let $G$ be a locally compact group, and let $\alpha: G \rightarrow \operatorname{Aut}(A)$ be a continuous action. Then the map

$$
\theta: L^{1}(G, A, \alpha) \rightarrow L^{1}\left(G, A^{\mathrm{op}}, \alpha^{\mathrm{op}}\right)
$$

given by $\theta(f)(s)=\Delta\left(s^{-1}\right) \alpha_{s}\left(f\left(s^{-1}\right)\right)$, for all $f \in L^{1}(G, A, \alpha)$ and all $s \in G$, is an isometric anti-isomorphism, which moreover extends to isometric anti-isomorphisms

$$
F^{p}(G, A, \alpha) \cong F^{p^{\prime}}\left(G, A^{\mathrm{op}}, \alpha^{\mathrm{op}}\right) \text { and } F_{\lambda}^{p}(G, X, \alpha) \cong F_{\lambda}^{p^{\prime}}\left(G, A^{\mathrm{op}}, \alpha^{\mathrm{op}}\right) \text {. }
$$

In particular, $F^{p}(G, A, \alpha)$ and $F_{\lambda}^{p}(G, A, \alpha)$ are anti-isometrically representable on $L^{p^{\prime}}$-spaces.

Proof. Be begin by showing that the map $\theta$ from the statement is an isometric anti-isomorphism.

That $\theta$ is isometric follows from the definition of $\Delta$. For $f, g \in L^{1}(G, A, \alpha)$ and $s \in G$, we have

$$
\theta(f * g)(s)=\Delta\left(s^{-1}\right) \alpha_{s}(f * g)\left(s^{-1}\right)=\Delta\left(s^{-1}\right) \alpha_{s}\left(\int_{G} f(t) \alpha_{t}\left(g\left(t^{-1} s^{-1}\right)\right) d t\right) .
$$


On the other hand, in the next computation we set $s^{-1} t=k$ at the fourth step to get

$$
\begin{aligned}
(\theta(g) * \theta(f))(s) & =\int_{G} \theta(g)(t) \cdot \text { op } \alpha_{t}^{\mathrm{op}}\left(\theta(f)\left(t^{-1} s\right)\right) d t \\
& =\int_{G} \alpha_{t}\left(\Delta\left(s t^{-1}\right) \alpha_{t^{-1} s}\left(f\left(s^{-1} t\right)\right) \Delta\left(t^{-1}\right) \alpha_{t}\left(g\left(t^{-1}\right)\right) d t\right. \\
& =\Delta\left(s^{-1}\right) \alpha_{s}\left(\int_{G} f\left(s^{-1} t\right) \alpha_{s^{-1} t}\left(g\left(t^{-1}\right)\right) d t\right) \\
& =\Delta\left(s^{-1}\right) \alpha_{s}\left(\int_{G} f(k) \alpha_{k}\left(g\left(k^{-1} s^{-1}\right)\right) d k\right),
\end{aligned}
$$

which proves the claim.

Denote by $\iota: G \rightarrow G$ the inversion map, which is anti-multiplicative. Let $\pi$ and $u$ be representations of $A$ and $G$, respectively, on an $L^{p}$-space $E$. By abuse of notation, we denote by $\pi^{\prime}: A \rightarrow \mathcal{B}\left(E^{\prime}\right)$ and $u^{\prime}: G \rightarrow \mathcal{B}\left(E^{\prime}\right)$ the homomorphisms given by $\pi^{\prime}(a)=\pi(a)^{\prime}$ and $u_{g}^{\prime}=\left(u_{g}\right)^{\prime}$ for all $a \in A$ and $g \in G$. Then $\pi^{\prime}$ and $u^{\prime} \circ \iota$ are representations of $A^{\text {op }}$ and $G$, respectively, on the $L^{p^{\prime}}$-space $E^{\prime}$ (and conversely, by reflexivity). Moreover, for $g \in G$ and $a \in A$, the identity $u_{g} \pi(a) u_{g^{-1}}=\pi\left(\alpha_{g}(a)\right)$ is equivalent to $u_{g^{-1}}^{\prime} \pi^{\prime}(a) u_{g}^{\prime}=\pi^{\prime}\left(\alpha_{g}(a)\right)$. It follows that $(\pi, u)$ is a covariant representation for $(G, A, \alpha)$ if and only if $\left(\pi^{\prime}, u^{\prime} \circ \iota\right)$ is a covariant representation for $\left(G, A^{\mathrm{op}}, \alpha^{\mathrm{op}}\right)$. This shows that the assignment $(\pi, u) \mapsto\left(\pi^{\prime}, u^{\prime} \circ \iota\right)$ induces a natural bijection between the classes $\operatorname{Rep}_{p}(G, A, \alpha)$ and $\operatorname{Rep}_{p^{\prime}}\left(G, A^{\mathrm{op}}, \alpha^{\mathrm{op}}\right)$. By the definition of the norm on the full crossed product, we conclude that

$$
\|f\|_{F^{p}(G, A, \alpha)}=\|\theta(f)\|_{F^{p^{\prime}}\left(G, A^{\mathrm{op}}, \alpha^{\mathrm{op}}\right)} .
$$

for all $f \in L^{1}(G, A, \alpha)$. This proves the statement for full crossed products.

The case of reduced crossed products follows similarly: the above bijection restricts to a bijection between $\operatorname{RegRep}_{p}(G, A, \alpha)$ and $\operatorname{Reg}_{\operatorname{Rep}}\left(G, A^{\mathrm{op}}, \alpha^{\mathrm{op}}\right)$, since for an $L^{p}$-space $E_{0}$, the transpose of the representation $\lambda_{p}^{E_{0}}$ is $\lambda_{p^{\prime}}^{E_{0}^{\prime}} \circ \iota$.

Note that if a Banach algebra $B$ is isometrically isomorphic to its opposite, then any Banach algebra completion of $B$ is also isomorphic to its opposite. We will use this (trivial) observation in the next corollary, with $B=L^{1}(G, A, \alpha)$ and the completions being the full and reduced crossed products.

Corollary 5.9. Adopt the notation of Proposition 5.8, and suppose that $A$ is abelian. Then $L^{1}(G, A, \alpha)$ is canonically isometrically isomorphic to $L^{1}(G, A, \alpha)^{\mathrm{op}}$, and moreover there are natural isometric isomorphisms

$$
F^{p}(G, A, \alpha) \cong F^{p^{\prime}}(G, A, \alpha) \quad \text { and } \quad F_{\lambda}^{p}(G, A, \alpha) \cong F_{\lambda}^{p^{\prime}}(G, A, \alpha) .
$$

Proof. Observe that $A$ is also an $L^{p^{\prime}}$-operator algebra, since it is abelian. By the first part of Proposition 5.8, and since $A$ is abelian, the map $\theta$ is a natural isometric isomorphism $L^{1}(G, A, \alpha) \cong L^{1}(G, A, \alpha)^{\text {op }}$. Upon taking completions with respect to all covariant representations of $(G, A, \alpha)$, we conclude that $F^{p}(G, A, \alpha)$ is isometrically isomorphic to its opposite algebra. Composing this isomorphism with the isomorphism $F^{p}(G, A, \alpha)^{\mathrm{op}} \cong F^{p^{\prime}}(G, A, \alpha)$ given by Proposition 5.8, we obtain the desired isometric isomorphism for full crossed products.

The case of reduced crossed products is identical: one completes with respect to regular covariant representations instead. 
The following is the main result of this section. When $X$ is the one point space, we recover Theorem 4.12. We point out that we do not know how to prove Theorem 5.10 directly without first obtaining some form of Theorem 4.12, and that Corollary 4.13 is not strong enough to deduce Theorem 5.10 from it.

Theorem 5.10. Let $X$ be a locally compact Hausdorff space, let $G$ be a nontrivial locally compact group, and let $\alpha: G \rightarrow \operatorname{Homeo}(X)$ be a topological action. Given $p, q \in[1, \infty)$ with $q>1$, the Banach algebras $F^{p}(G, X, \alpha)$ and $F_{\lambda}^{p}(G, X, \alpha)$ can be isometrically represented on an $L^{q}$-space if and only if one of the following holds:

(1) $\left|\frac{1}{p}-\frac{1}{2}\right|=\left|\frac{1}{q}-\frac{1}{2}\right|$; or

(2) $G$ is abelian, $p=2$ and the action $\alpha$ is trivial.

Proof. If $\left|\frac{1}{p}-\frac{1}{2}\right|=\left|\frac{1}{q}-\frac{1}{2}\right|$, then the conclusion follows from Corollary 5.9. Suppose that $p=2$, that $G$ is abelian and that the action is trivial. Denote by $\widehat{G}$ the dual group of $G$. Then one easily checks that $F_{\lambda}^{2}(G, X, \alpha)$ and $F^{2}(G, X, \alpha)$ are both isometrically isomorphic to $C_{0}(X \times \widehat{G})$, so they are representable on an $L^{q}$-space, for any $q \in[1, \infty)$. This proves the "if" implication.

Let us show the converse. We treat the case of reduced crossed products first. Let $p, q \in[1, \infty)$ with $q>1$, let $E$ be an $L^{q}$-space and let $\varphi: F_{\lambda}^{p}(G, X, \alpha) \rightarrow \mathcal{B}(E)$ be an isomeric isomorphism. Since $C_{0}(X)$ has a (two-sided) contractive approximate identity, so does $F_{\lambda}^{p}(G, X, \alpha)$ by Theorem 5.5. Hence, upon restricting to its essential subspace and using Theorem 2.2 we may assume that $\varphi$ is nondegenerate. Let $\widetilde{\varphi}: M\left(F_{\lambda}^{p}(G, X, \alpha)\right) \rightarrow \mathcal{B}(E)$ be the extension of $\varphi$ provided by Theorem 4.5. By Theorem 5.6, there exists a canonical isometric homomorphism $\iota_{\lambda}^{G}: F_{\lambda}^{p}(G) \rightarrow M\left(F_{\lambda}^{p}(G, X, \alpha)\right)$. We conclude that $\widetilde{\varphi} \circ \iota_{\lambda}^{G}$ is an isometric representation of $F_{\lambda}^{p}(G)$ on an $L^{q}$-space. By Theorem 4.12, either $\left|\frac{1}{p}-\frac{1}{2}\right|=\left|\frac{1}{q}-\frac{1}{2}\right|$ or $p=2$ and $G$ is abelian. Assuming the latter, $F^{2}(G, X, \alpha)$ is a $C^{*}$-algebra. Suppose, without loss of generality, that $q \neq 2$. Then $F^{2}(G, X, \alpha)$, and thus its multiplier algebra $M\left(F^{2}(G, X, \alpha)\right)$, must be abelian by Theorem 4.4 For $g \in G$, let $u_{g} \in M\left(F^{2}(G, X, \alpha)\right)$ denote the canonical unitary implementing $\alpha_{g}$. For $a \in C_{0}(X) \subseteq M\left(F^{2}(G, X, \alpha)\right)$, we have

$$
\alpha_{g}(a)=u_{g} a u_{g}^{*}=a .
$$

It follows that $\alpha$ is trivial, as desired. This shows the statement for $F_{\lambda}^{p}(G, X, \alpha)$.

We prove the statement for full crossed products now. Let $p, q \in[1, \infty)$ with $q>1$, let $F$ be an $L^{q}$-space and let $\psi: F^{p}(G, X, \alpha) \rightarrow \mathcal{B}(F)$ be an isomeric isomorphism. As before, we may assume that $\psi$ is nondegenerate, and we denote by $\widetilde{\psi}: M\left(F^{p}(G, X, \alpha)\right) \rightarrow \mathcal{B}(F)$ its unital extension. Let $g \in G \backslash\{1\}$, and denote by $H \leq G$ the (not necessarily closed) cyclic subgroup of $G$ generated by $g$. Then $H$ is amenable and there is a commutative diagram of contractive homomorphisms (explanations follow below)

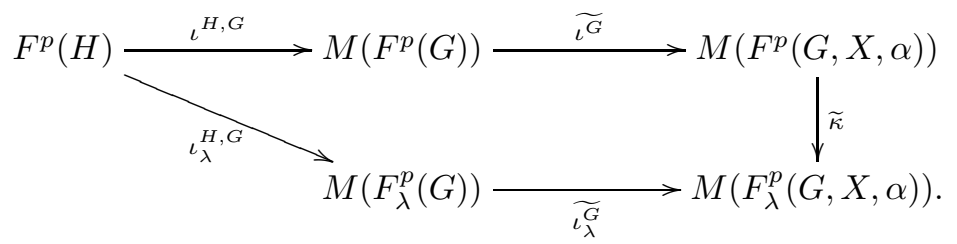


In the diagram above, $\iota^{H, G}$ and $\iota_{\lambda}^{H, G}$ are the canonical isometric inclusions provided by Remark 4.8 and Proposition 4.9 $\widetilde{\iota^{G}}$ and $\widetilde{\iota_{\lambda}^{G}}$ are the canonical unital extensions of the maps constructed in Theorem 5.6 and $\widetilde{\kappa}$ is the unital extension of the canonical map $\kappa$ from full to reduced crossed product. Since $\iota_{\lambda}^{G}$ is isometric by Theorem 5.6, so is $\widetilde{\iota_{\lambda}^{G}}$. By commutativity of the diagram, $\widetilde{\kappa} \circ \widetilde{\iota}^{G} \circ \iota^{H, G}$ is an isometric representation of $F^{p}(H)$ on the $L^{q}$-space $F$. By Theorem 4.12, we must have either $\left|\frac{1}{p}-\frac{1}{2}\right|=$ $\left|\frac{1}{q}-\frac{1}{2}\right|$, or $p=2$. Assume that $p=2$ and $q \neq 2$. Then $F^{p}(G, X, \alpha)$ is a $C^{*}$-algebra, so it must be abelian by Theorem 4.4 Since $C_{\lambda}^{*}(G)$ embeds into the abelian $C^{*}$ algebra $M\left(F_{\lambda}^{2}(G, X, \alpha)\right)$, the group $G$ itself must be abelian. Finally, the same argument used before shows that $\alpha$ must be trivial. This finishes the proof.

Finally, the following corollary asserts that the $L^{p}$-crossed products obtained from topological dynamical systems, for varying $p$, are pairwise non-isometrically isomorphic, except for conjugate exponents.

Corollary 5.11. Let $X$ be a locally compact Hausdorff space, let $G$ be a locally compact group, and let $\alpha: G \rightarrow \operatorname{Homeo}(X)$ be a topological action. Given $p, q \in$ $[1, \infty)$, the following conditions are equivalent:

(1) $F^{p}(G, X, \alpha)$ is isometrically isomorphic to $F^{q}(G, X, \alpha)$;

(2) $F_{\lambda}^{p}(G, X, \alpha)$ is isometrically isomorphic to $F_{\lambda}^{q}(G, X, \alpha)$;

(3) $\left|\frac{1}{p}-\frac{1}{2}\right|=\left|\frac{1}{q}-\frac{1}{2}\right|$.

\section{REFERENCES}

[BLM95] D. Blecher and C. Le Merdy, On quotients of function algebras and operator algebra structures on $\ell_{p}$, J. Operator Theory 34 (1995), no. 2, 315-346.

[Bon54] F. Bonsall, A minimal property of the norm in some Banach algebras, J. London Math. Soc. 29(1954), 156-164.

[Cow98] M. Cowling, The predual of the space of convolutors on a locally compact group, Bull. Austral. Math. Soc. 57 (1998), 409-414.

[Dal00] G. Dales, Banach Algebras and Automatic Continuity, London Mathematical Society Monographs, New Series, no. 24, The Clarendon Press, Oxford University Press, New York, 2000.

[DS13] M. Daws and N. Spronk, The approximation property implies that convolvers are pseudomeasures, Preprint. (arXiv:1308.1073 [math.FA]), 2013.

[GT14] , Functoriality of group algebras acting on $L^{p}$-spaces, preprint, arXiv:1408.6137, 2014.

[GT15a] E. Gardella and H. Thiel, Banach algebras generated by an invertible isometry of an $L^{p}$-space. J. Funct. Anal. 269 (2015), 1796-1839.

[GT15b] _ Group algebras acting on $L^{p}$-spaces, J. Fourier Anal. Appl. 21 (2015), 13101343.

[GT16a] _ Quotients of Banach algebras acting on $L^{p}$-spaces, Adv. Math. 296 (2016), 85-92.

[GT16b] - Isomorphisms of algebras of convolution operators, in preparation, 2016.

[GT16c] _ Extensions of representations of Banach algebras to biduals, in preparation, 2016.

[Her73] C. Herz, Harmonic synthesis for subgroups, Ann. Inst. Fourier (Grenoble) 23 (1973), 91-123.

[Lam58] J. Lamperti, On the isometries of certain function-spaces, Pacific J. Math. 8 (1958), $459-466$.

[NR09] M. Neufang and V. Runde, Column and row operator spaces over $\mathrm{QSL}_{p}$-spaces and their use in abstract harmonic analysis, preprint, arxiv:0711.2057, 2007. 
[Phi13] N. C. Phillips, Crossed products of $L^{p}$ operator algebras and the K-theory of Cuntz algebras on $L^{p}$ spaces, preprint, arXiv:1309.6406, 2013.

[Phi14] _ Multiplicative domain for $L^{p}$ operator algebras, in preparation. Draft of May 2014

[Run05] V. Runde, Representations of locally compact groups on $\mathrm{QSL}_{p}$-spaces and a p-analog of the Fourier-Stieltjes algebra, Pacific J. Math. 221 (2005), 379-397.

[Spa12] P. Spain, Representations of $C^{*}$-algebras in dual $\mathcal{E}$ right dual Banach algebras. Houston J. Math. 41 (2015), no. 1, 231-263.

[Tza69] L. Tzafriri, Remarks on contractive projections in $L_{p}$-spaces. Israel J. Math. 71969 9-15.

Eusebio Gardella Mathematisches Institut, Fachbereich Mathematik und Informatik Der Universität Münster, Einsteinstrasse 62, 48149 Münster, Germany.

E-mail address: gardella@uni-muenster.de

$U R L$ : www .math.uni-muenster.de/u/gardella/

Hannes Thiel Mathematisches Institut, Fachbereich Mathematik und Informatik der Universität Münster, Einsteinstrasse 62, 48149 Münster, Germany.

E-mail address: hannes.thiel@uni-muenster.de

$U R L$ : www .math. uni-muenster.de/u/hannes.thiel/ 